\title{
SARS-CoV-2 variant of concern type and biological sex affect efficacy of molnupiravir in dwarf hamster model of severe COVID-19
}

Carolin M. Lieber ${ }^{1 \#}$, Robert M Cox ${ }^{1 \#}$, Julien Sourimant ${ }^{1}$, Josef D. Wolf ${ }^{1}$, Kate Juergens ${ }^{2}$, Quynh Phung ${ }^{2}$,

Manohar T Saindane ${ }^{3}$, Michael G Natchus ${ }^{3}$, George R Painter ${ }^{3}$, Kaori Sakamoto ${ }^{4}$, Alexander L. Greninger ${ }^{2}$, Richard K Plemper ${ }^{1 *}$

${ }^{1}$ Center for Translational Antiviral Research, Institute for Biomedical Sciences, Georgia State University, Atlanta, GA 30303

${ }^{2}$ Virology Division, Department of Laboratory Medicine, University of Washington, Seattle, WA 98185

${ }^{3}$ Emory Institute for Drug Development, Emory University, Atlanta, GA 30322

${ }^{4}$ Department of Pathology, College of Veterinary Medicine, University of Georgia, Athens, GA 30602

${ }^{\#}$ these authors contributed equally to this work

Short title: VOC type and sex affect efficacy of molnupiravir

*to whom correspondence should be addressed: rplemper@gsu.edu 


\section{Summary Paragraph}

SARS-CoV-2 variants of concern (VOC) have triggered distinct infection waves in the coronavirus disease

32019 (COVID-19) pandemic, culminating in currently all-time high incidence rates of VOC omicron. Orally

4 available direct-acting antivirals such as molnupiravir promise to improve disease management and limit SARS-

5 CoV-2 spread. However, molnupiravir efficacy against VOC delta was questioned based on clinical trial results

6 and its potency against omicron is unknown. This study evaluates molnupiravir against a panel of relevant VOC

7 in three efficacy models: primary human airway epithelium organoids, the ferret model of upper respiratory

8 disease, and a lethal Roborovski dwarf hamster efficacy model of severe COVID-19-like acute lung injury. All

9 VOC were equally efficiently inhibited by molnupiravir in cultured cells and organoids. Treatment consistently

0 reduced upper respiratory VOC shedding in ferrets and prevented viral transmission. Pathogenicity in the dwarf

1 hamsters was VOC-dependent and highest for gamma, omicron, and delta with fulminant lung histopathology.

2 Oral molnupiravir started 12 hours after infection resulted in complete survival of treated dwarf hamsters

3 independent of challenge VOC. However, reduction in lung virus differed VOC-dependently, ranging from one

4 (delta) to four (gamma) orders of magnitude compared to vehicle-treated animals. Dwarf hamsters infected with

5 VOC omicron showed significant individual variation in response to treatment. Virus load reduction was

6 significant in treated males, but not females. The dwarf hamster model recapitulates mixed efficacy of

7 molnupiravir seen in human trials and alerts that therapeutic benefit of approved antivirals must be continuously

8 reassessed in vivo as new VOC emerge. 
By February 2022, SARS-CoV-2 has resulted in over 385 million cases and more than 5.7 million deaths worldwide $^{1}$. Vaccines are widely available ${ }^{2,3}$, but limited longevity of vaccine-induced immunity, hesitancy of 2 population subgroups to vaccinate ${ }^{4,5}$, and increasingly contagious and/or vaccine-insensitive variants of concern

3 (VOC) alpha (B.1.1.7 lineage), beta (B.1.351 lineage), gamma (P.1 lineage), delta (B.1.617.2 lineage), and 4 omicron (B1.1.529 lineage $)^{6,7}$ have fueled recuring global infection waves. VOC delta was the prevalent 5 circulating variant during Summer and Fall 2021 due to replication to high titers, prolonged shedding from 6 infected individuals, and a propensity to induce breakthrough-infections in vaccinees ${ }^{8-10}$. Since its first appearance 7 in November 2021, VOC omicron has rapidly replaced delta as the dominant circulating strain in most 8 geographical regions ${ }^{11}$, propelled by sharply reduced sensitivity to neutralizing antibodies directed against earlier 9 lineages and greatly increased infectivity ${ }^{12}$. Although clinical signs associated with VOC omicron are typically 0 milder than those of its predecessors, record-high daily infection rates have driven high absolute hospitalization 1 numbers, creating an urgent need for therapeutics to improve disease management.

Molnupiravir was the first orally available SARS-CoV-2 inhibitor approved for clinical use against COVID$19^{13}$. Intermediate results of the early months of a large efficacy trial revealed an encouraging $50 \%$ reduction of hospitalizations in the treatment group, but later analysis of the full dataset reduced efficacy to a $30 \%$ lower hospitalization rate overall ${ }^{14}$. Based on geographical location of trial participants and VOC prevalence in the earlier versus later phase of the trial, an advisory board to the FDA considered lower efficacy of molnupiravir against VOC delta as a possible explanation for the mixed results ${ }^{15}$. However, VOC delta was efficiently inhibited by the molnupiravir parent metabolite $\mathrm{N}^{4}$-hydroxycytidine $(\mathrm{NHC})$ in ex vivo studies ${ }^{16}$, suggesting unchanged sensitivity to the drug.

Human airway epithelium organoids, ferrets, mice, and Syrian golden hamsters have emerged as preclinical models to assess efficacy of anti-SARS-CoV-2 drug candidates. Ferrets recapitulate the predominant clinical presentation of SARS-CoV-2 in younger patients, characterized by high viral load in the upper respiratory tract, strong viral shedding, and efficient airborne transmission ${ }^{17-19}$. By contrast, Syrian golden hamsters infected with 
5 COVID-19. Disease typically remains mild-to-asymptomatic in golden hamsters and animals fully recover within

6 two weeks ${ }^{20}$. Lethal disease with severe histopathology affecting lung, liver, and kidney can be induced in

7 transgenic K18-hACE2 mice expressing human ACE2, but organ distribution of the receptor is non-physiological,

8 resulting in rapid neuro-invasion of the virus and the development of acute, lethal viral encephalitis ${ }^{21}$, which is

9 not seen in human patients. Missing is an efficacy model that recapitulates the acute lung injury of life-threatening

0 COVID-19, thereby offering a relevant experimental platform to test the effect of molnupiravir on mitigating lung

1 damage caused by different VOC and explore the impact of treatment on disease outcome. We selected

2 Roborovski dwarf hamsters as a candidate model for this study, since these dwarf hamsters developed acute

3 diffuse alveolar pneumonia and succumbed to infection ${ }^{22,23}$ when challenged with original SARS-CoV-2 isolates

4 from 2020.

For quantitative comparison of molnupiravir activity against VOC alpha, beta, gamma, delta and omicron in

6 cultured cells, we determined half-maximal antiviral concentrations $\left(\mathrm{EC}_{50} \mathrm{~s}\right)$ on Vero-E6 cells stably expressing

7 TMPRSS2 (Vero-TMPRSS2) (Fig. 1a). The molnupiravir parent compound NHC was used in all ex vivo

8 experiments. Inhibitory values were similar for all VOC tested and ranged from 0.27 (omicron) to 0.62 (alpha)

$9 \mu \mathrm{M}$, which closely resembled that against the original SARS-CoV-2 isolate ${ }^{18,24}$. Consistent with other cell culture

$0 \quad$ data $^{16}$, also VOC delta was highly sensitive to treatment $\left(\mathrm{EC}_{50} 0.33 \mu \mathrm{M}\right)$.

\section{Consistent activity of molnupiravir against VOC in human airway organoids}

To validate activity in disease-relevant primary human tissues, we added $\mathrm{NHC}$ at $10 \mu \mathrm{M}$, equivalent to $\mathrm{NHC}$

3 plasma concentrations reached in human patients ${ }^{25}$, to the basolateral chamber of well-differentiated primary human airway epithelium (HAE) cultures grown at air-liquid interface. After infection with the panel of five VOC, we titered apically shed progeny virions after 1,2 , and 3 days. Treatment was instantly sterilizing in the case of VOC alpha, beta and delta, and within 2 days reduced progeny load of all VOC to level of detection (Fig. 1b). Parallel assessment of transepithelial electrical resistance (TEER) as indicator of an intact tight junction network demonstrated that the drug preserved the integrity of the infected organoids (Extended Data 1). Vehicletreated controls showed significant reductions in TEER after infection, reflecting breakdown of epithelium 
1 abundant viral antigen (SARS-CoV-2 nucleocapsid and spike protein) was detected in vehicle treated epithelia

2 (Fig. 1c, Supplementary Fig. 1,2).

\section{Efficacy of molnupiravir against VOC upper respiratory disease and transmission in ferrets}

To assess in vivo efficacy of molnupiravir against VOC, we infected ferrets intranasally with $1 \times 10^{5} \mathrm{pfu}$ of

VOC alpha, beta, gamma, delta, and omicron and monitored virus replication in the upper respiratory tract. All

treated animals were dosed orally at $5 \mathrm{mg} / \mathrm{kg}$ b.i.d., starting 12 hours after infection (Fig. 2a) when shed SARS-

CoV-2 becomes first detectable in ferret nasal lavages ${ }^{18,19}$. Lavage titers were determined in 12-hour intervals for

the first 48 hours after infection, and once daily thereafter. Titers of shed VOC alpha, gamma, delta, and omicron

peaked 1 to 2 days after infection at approximately $10^{3}$ to $10^{4} \mathrm{pfu}$ per $\mathrm{ml}$ in vehicle-treated animals (Fig. 2b).

Treatment with molnupiravir reduced shed progeny titers of all VOC to detection level within 12 hours. Consistent

with previous experience with the ferret model ${ }^{18,19}$, infected animals developed no clinical signs. VOC beta did

not establish a productive infection and was eliminated from further ferret experiments (Supplementary Fig. 3).

To explore the impact of treatment on transmission, we co-housed infected and treated source animals with

uninfected and untreated sentinels for 48 hours, starting 42 hours after initiation of treatment (Fig. 2c). VOC delta

was excluded from transmission studies, since we had not detected any infectious particles in nasal lavages of

molnupiravir-treated animals at any time and nasal turbinates of treated animals extracted four days after infection

did not contain any infectious particles (Extended Data 2). We tested VOC alpha in an independent transmission study, but had included transmission arms in the efficacy studies with VOC gamma and omicron (Fig. 2b) to

reduce overall animal numbers. After separation of source and contact animals on day 4 after infection, untreated

sentinels were monitored for an additional four days for shed virions and viral RNA in nasal lavages and then

1 terminal titers in nasal turbinates determined.

VOC alpha and gamma spread efficiently from vehicle-treated source animals to the sentinels (Fig. 2d,e), whereas VOC omicron did not transmit (Fig. 2f). RNA of VOC alpha and gamma became first detectable in nasal lavages of the contacts within 12 hours after initiation of co-housing, shed infectious particles emerged after 12 (VOC alpha) to 36 (VOC gamma) hours (Fig. 2e,f), and infectious alpha and gamma, but not omicron, particles 
7 (Extended Data 3). Treatment with molnupiravir fully suppressed transmission. No infectious particles or viral

8 RNA were detectable in lavages of untreated sentinels, and nasal turbinates of these animals were virus and viral

9 RNA-free at terminal assessment (Fig 2e,f; Extended Data 3). These data demonstrate that oral molnupiravir is

0 highly effective in controlling replication of all VOC in the ferret upper respiratory tract, significantly reducing

1 shed virus titers and rapidly suppressing spread of transmission-competent VOC to untreated naïve contacts.

2 Different degrees of acute lung pathogenesis of VOC in Roborovski dwarf hamsters

3 To explore VOC pathogenicity in a candidate model of lethal COVID-19, we infected Roborovski dwarf

4 hamsters intranasally with $1 \times 10^{5}$ pfu each of the original SARS-CoV-2 WA1 isolate or VOC alpha, beta, gamma,

5 and omicron, or $3 \times 10^{4} \mathrm{pfu}$ of VOC delta (Fig. 3a) and monitored clinical signs and survival. Dwarf hamsters

6 showed a rapid decline characterized by ruffled fur, lethargy, and dyspnea within two days of infection, which

17 was accompanied by hypothermia and moderate to substantial loss of body weight (Fig. 3b,c). Severity and time

8 to onset of clinical signs varied between - in the order of increasing pathogenicity - VOC alpha, beta, omicron,

9 gamma, and delta. Pathogenesis of VOC gamma was comparable to that of the original WA1 isolate. Median

0 survival of animals infected with WA1 and VOC beta, gamma, delta, and omicron was shortened to 5.75 (WA1),

16 (omicron), 3.5 (beta, gamma) and 3 (delta) days after infection (Fig 3d; Extended Data 4). Approximately 80\%

2 of animals inoculated with VOC alpha survived the infection, making alpha the least lethal of the VOC tested.

3 We intended to determine viral tissue tropism and gross lung pathology three days after infection of subsets

4 of hamsters with WA1 or the VOC associated with the overall highest mortality rate, gamma, delta and omicron.

5 However, animals infected with delta succumbed to infection before the predefined endpoint, preventing analysis.

6 Animals infected with WA1, gamma, or omicron consistently showed the highest viral RNA load in lung,

7 followed by small and large intestine and spleen (Fig. 3e). Macroscopic analysis of extracted lungs showed

8 substantial gross tissue damage involving large surface areas (Fig. 3f, Supplementary Fig. 4) and titration of lung

9 homogenates confirmed fulminant viral pneumonia with median virus load of $1 \times 10^{5} \mathrm{pfu} / \mathrm{g}$ lung tissue or greater

0 (Fig. 3g). A follow-up study with VOC delta at reduced inoculum amount $\left(1 \times 10^{4}\right.$ pfu intranasally) and shortened

1 time to analysis (Fig. 3h) demonstrated onset of lung tissue damage (Fig. 3i, Supplementary Fig. 5) and presence 
2 of high lung virus loads (Fig. 3j) already 12 hours after infection. Comparison analysis of VOC gamma 24 hours

3 after infection confirmed that rapid viral invasion of the dwarf hamsters was not limited to VOC delta.

4 Effect of VOC type and biological sex on prevention of lethal viral pneumonia

VOC gamma, delta, and omicron were selected for an efficacy study assessing mitigation of viral pneumonia

6 and acute lung injury with molnupiravir. Animals were inoculated with $1 \times 10^{4}$ pfu intranasally to prevent

7 premature death, followed by initiation of treatment ( $250 \mathrm{mg} / \mathrm{kg}$ orally b.i.d.) 12 hours after infection (Fig. $4 \mathrm{a})$,

8 when lung virus load was high and first lung lesions became detectable (Fig. 3i,j). The higher molnupiravir dose

9 compared to that administered to ferrets was used to compensate for high metabolic activity of the dwarf

0 hamsters $^{27}$ and is consistent with the dose level administered to other rodent species ${ }^{28,29}$. Oral molnupiravir

1 alleviated clinical signs (Extended Data 5) and ensured complete survival of all treated animals independent of

2 challenge VOC, whereas approximately $50 \%$ (gamma) and $90 \%$ (delta, omicron) of animals in the different

3 vehicle groups succumbed to the infection within 2 to 7 days (Fig. 4b). Lung virus load assessed three days after

4 infection was consistently high (approximately $10^{7}-10^{8} \mathrm{pfu} / \mathrm{g}$ lung tissue) in all vehicle-treated groups (Fig. $4 \mathrm{c}$ ).

5 Treatment significantly lowered lung titers independent of challenge VOC, albeit effect size varied greatly from

6 approximately 1 (delta) to over 4 (gamma) orders of magnitude. Although likewise statistically significant

7 compared to vehicle-treated animals, impact of molnupiravir on omicron lung load showed major variation

8 between individual animals, creating low, high, and super responder groups characterized by lung titer reductions

9 of approximately 1, 4, and $>5$ orders of magnitude, respectively (Fig. 4c). Infectious titers in lung were closely

$0 \quad$ mirrored by viral RNA copies present in lung and tracheas (Extended Data 6).

1 Having powered this study with approximately equal numbers of male and female animals in each group, we

2 queried the dataset for a possible impact of biological sex on outcome (Fig. 4d). No significant differences in lung

3 virus load between males and females were detected in any of the three vehicle groups, which was consistent with

4 absence of a correlation between biological sex and probability of survival of the vehicle-treated animals in our

5 survival study (Extended Data 7). Comparison of lung virus burden of males and females in the molnupiravir-

6 treated groups revealed no statistically significant differences in effect size in animals infected with VOC gamma

7 or delta, but biological sex had a statistically significant influence on molnupiravir benefit of animals infected 
8 with omicron (Fig. 4d, Extended Data 8). Whereas lung titer reductions in treated males were highly significant

9 compared to vehicle-treated males, females, or all vehicle-treated animals combined, no significant change in

$0 \quad$ lung virus load against any of these vehicle groups was detected in females treated with molnupiravir.

1 Species-adaptation of SARS-CoV-2 VOC in dwarf hamsters

3 adaptation mutations such as an L260F substitution in nsp6 and a Y453F mutation in spike ${ }^{26}$ in virus populations

4 extracted from ferret nasal turbinates. To probe for possible virus adaptations to the dwarf hamsters, we sequenced

5 whole genomes of virus populations recovered from the different vehicle or molnupiravir treatment groups. No

6 dwarf hamster-typical mutations were detected that were dominant across all VOC populations, but we detected

7 several VOC type-specific substitutions with $>20 \%$ allele frequency compared to the respective virus inoculum.

8 Irrespective of treatment status, a spike D142G substitutions was present in nearly all VOC delta populations, but

9 no dominating mutations emerged in spike proteins of the different VOC gamma and omicron reisolates

0 (Supplementary Table 1). All recovered VOC gamma populations harbored an nsp6 V181F substitution and all

1 VOC omicron populations contained the nsp6 L260F mutation that was originally considered to be characteristic

2 for adaptation to weasels ${ }^{19}$. However, none of the recovered VOC delta populations contained substitutions in

3 nsp6. We found isolated additional substitutions in some virus populations recovered from individual animals in

4 the respective infection and treatment groups (Supplementary Table 1, Supplementary Datasets 1-3), but detected

5 no correlation to relative viral fitness in vehicle-treated dwarf hamsters or link to molnupiravir treatment success.

\section{Molnupiravir-mediated mitigation of lung histopathology}

Macroscopic assessment of the lungs extracted three days after infection revealed severe tissue damage with

large lesions covering approximately $30 \%$ (omicron) to $50 \%$ (gamma, delta) of the lung surface area of vehicletreated animals (Fig. 4e, Supplementary Fig. 6). Molnupiravir treatment significantly reduced macroscopic tissue

damage independent of challenge VOC (Fig. 4e, Extended Data 9). Histological examination of lungs extracted from animals infected with VOC gamma and delta revealed markers of severe viral infection in vehicle-treated animals, including perivascular cuffing, alveolitis, hyalinization of blood vessels, interstitial pneumonia, and leucocyte infiltration (Fig. 4f, Extended Data 9). One of the VOC gamma-infected animals developed pronounced 
4 peribronchiolar metaplasia. Due to high lethality of VOC delta, only one animal of the vehicle group reached the

5 predefined endpoint for tissue harvest in this study, whereas the others died prematurely and could not be

6 examined. Molnupiravir alleviated histopathology associated with either VOC, decreasing immune cell

7 infiltration and reducing signs of inflammation. Greater residual damage was detected in treated animals infected

8 with VOC delta compared to gamma, which was consistent with the significantly greater molnupiravir-mediated

9 reduction in gamma lung load detected in the efficacy study (Fig. 4c).

0 Immunohistochemistry analysis of the lung sections identified abundant viral antigen in animals of the

1 vehicle-treated groups (Fig. 4g). Lung sections of molnupiravir-treated animals returned variable results

2 depending on VOC, ranging from strong staining after infection with delta to complete absence of viral antigen

3 after VOC gamma. This differential staining intensity recapitulated the differences observed in lung virus load

4 and viral RNA copies after treatment of animals infected with VOC delta versus gamma (Fig. 4c, Extended Data

5 6). When we examined lung sections of molnupiravir-treated animals and surviving members of the vehicle

6 groups two weeks after infection, no viral antigen was detectable and only minor signs of infection were visible

7 (Extended Data 10), indicating that histopathological damage was transient in survivors.

8 These results demonstrate that acute lung injury occurs rapidly in the dwarf hamster model. Molnupiravir

9 consistently improves clinical signs and overall disease outcome independent of infecting VOC. However, effect

0 size of lung virus load reduction is greatly affected by VOC type and, in the case of infection with VOC omicron, 1 influenced by biological sex of the treated animal.

\section{Discussion}

of life-threatening viral pneumonia. We show that the dwarf hamsters provide a robust experimental system to instance, higher rates of severe disease and hospitalization associated with delta ${ }^{30-32}$ than omicron ${ }^{33}$. The 
0 i) Direct antiviral potency of the molnupiravir parent NHC against all VOC including delta and omicron was

1 virtually identical in cultured cells and human organoids, and closely resembled that reported for the original

2 WA1 isolate ${ }^{18}$. We consider this outcome unsurprising since extensive past attempts to resistance profile

3 molnupiravir through adaptation of different viral targets had remained unsuccessful ${ }^{34-36}$. Based on a high genetic

4 barrier preventing viral escape and the broad antiviral indication spectrum of molnupiravir ${ }^{18,35,37}$, there is no

5 molecular rationale why genetically closely related SARS-CoV-2 VOC that have no history of prior molnupiravir

6 exposure should be inherently less sensitive to the drug.

ii) Treatment with molnupiravir significantly reduced VOC shedding from the upper respiratory tract of

8 ferrets, shortening the time in which a host is infectious. Accordingly, molnupiravir suppressed transmission to

9 untreated sentinels. No VOC-specific differences in drug efficacy were apparent. However, all VOC, but gamma,

0 also grew to lower titers in the ferret upper respiratory tract than WA $1^{18}$ and omicron did not transmit at all. By

1 contrast, original SARS-CoV-2 isolates spread readily between ferrets through contact and airborne

2 transmission $^{38}$ and direct and reverse zoonotic transmission in the field have been reported for closely related

3 minks $^{39}$. Presumably reflecting increasing SARS-CoV-2 adaptation to the human host, this reduced ferret

4 permissiveness for SARS-CoV-2 VOC interfered with a meaningful comparison analysis of treatment efficacy.

iii) It was speculated that the variable clinical performance of molnupiravir in the interim versus complete

6 trial analysis could be attributable to lower effectiveness of the drug against VOC delta ${ }^{15}$. Delta indeed surged

7 only when the trial was underway and a large subgroup of trial participants was enrolled from the South American

8 region $^{14}$, in which gamma happened to be dominant until Fall 2021. By revealing a strong correlation between

9 effect size of molnupiravir efficacy and VOC type under controlled experimental conditions, the dwarf hamster

0 model suggests VOC-dependent therapeutic benefit as a tangible explanation for the clinical conundrum. At

1 present we cannot conclusively address, however, whether the difference in virus load reduction between VOC

2 gamma and delta in dwarf hamsters recapitulates the variable clinical success of molnupiravir because of a

3 common mechanism or due to a phenocopy effect. Although VOC delta and gamma replicated to similar lung

4 titers in vehicle-treated animals, delta was associated with the shortest time-to-death. Individual VOC could

5 indirectly modulate molnupiravir pharmacokinetic properties differentially by spreading to organs other than lung 
6 with distinct kinetics. However, our analysis of viral organ distribution in the dwarf hamsters revealed very low

7 viral RNA burden in liver, the primary site of drug metabolism ${ }^{40}$. Alternatively, the rapid-onset lung

8 histopathology seen with VOC delta may directly contribute to lower effect size of therapy.

iv) Unexpectedly, molnupiravir efficacy against VOC omicron was variable between individual dwarf

0 hamsters. Biological sex of the animals emerged as a correlate for therapeutic benefit of molnupiravir use against

1 omicron, with treated males faring overall better than females. By contrast, biological sex had no effect on

2 treatment benefit when dwarf hamster were infected with VOC gamma or delta, which matched human trial data

3 reported for these $\mathrm{VOC}^{14}$. Dwarf hamsters are outbred and animals used in this study were not raised under

4 controlled conditions, introducing individual differences in body weight, age, microbiome, drug metabolism,

5 and/or prior disease history as additional variables, which certainly are all equally present also in human patients.

6 However, dwarf hamsters were randomly assigned to the different study groups and these factors, if indeed of

7 importance, should have resulted in equal individual variation in viral load in the vehicle group or in animals

8 infected with VOC gamma or delta. Whole genome sequence analysis of VOC omicron populations recovered

9 from the hamsters at the end of infection revealed furthermore no correlation between potential differential VOC

0 omicron adaptation to the dwarf hamster host and effect size of molnupiravir therapy, pointing overall to high

1 variability of omicron disease dynamics in treated dwarf hamsters.

In the absence of controlled clinical data assessing molnupiravir efficacy against omicron, it is currently

3 unclear to what degree the dwarf hamster-derived results extend to human therapy. Our study demonstrates,

4 however, that pharmacological mitigation of severe COVID-19 is complex and that attempts to predict drug

5 efficacy based on unchanged ex vivo inhibitory concentrations alone ${ }^{16}$ may be premature. The dwarf hamster-

6 based results alert to the need to continuously reassess therapeutic benefit of approved antivirals for individual

7 patient subgroups as SARS-CoV-2 evolves and potential future VOC may emerge.

\section{$9 \quad$ Main References}

$0 \quad 1 \quad$ WHO. WHO Coronavirus (COVID-19) Dashboard, (https://covid19.who.int) (2021).

12 Polack, F. P. et al. Safety and Efficacy of the BNT162b2 mRNA Covid-19 Vaccine. N. Engl. J. Med. 383, 2 2603-2615, doi:10.1056/NEJMoa2034577 (2020). 
33 Jackson, L. A. et al. An mRNA Vaccine against SARS-CoV-2 - Preliminary Report. N Engl J Med 383, 1920-1931, doi:10.1056/NEJMoa2022483 (2020). 35, 775-779, doi:10.1007/s10654-020-00671-y (2020).

Fisher, K. A. et al. Attitudes Toward a Potential SARS-CoV-2 Vaccine : A Survey of U.S. Adults. Ann Intern Med 173, 964-973, doi:10.7326/M20-3569 (2020). Garcia-Beltran, W. F. et al. Multiple SARS-CoV-2 variants escape neutralization by vaccine-induced humoral immunity. Cell 184, 2372-2383 e2379, doi:10.1016/j.cell.2021.03.013 (2021). sera. Cell 184, 2348-2361 e2346, doi:10.1016/j.cell.2021.02.037 (2021).

Mlcochova, P. et al. SARS-CoV-2 B.1.617.2 Delta variant replication and immune evasion. Nature 599, 114-119, doi:10.1038/s41586-021-03944-y (2021).

10 Planas, D. et al. Reduced sensitivity of SARS-CoV-2 variant Delta to antibody neutralization. Nature 596, 276-280, doi:10.1038/s41586-021-03777-9 (2021).

11 Kupferschmidt, K. \& Vogel, G. Omicron threat remains fuzzy as cases explode. Science 375, 9-10, doi:10.1126/science.acz9928 (2022).

12 Perez-Then, E. et al. Neutralizing antibodies against the SARS-CoV-2 Delta and Omicron variants following heterologous CoronaVac plus BNT162b2 booster vaccination. Nat. Med., doi:10.1038/s41591022-01705-6 (2022).

13 GOV.UK First oral antiviral for COVID-19, Lagevrio (molnupiravir), approved by MHRA https://www.gov.uk/government/news/first-oral-antiviral-for-covid-19-lagevrio-molnupiravir-approvedby-mhra. (2021).

14 Molnupiravir - US Food and Drug Administration (https://www.fda.gov/media/154472/download). (2021).

15 Kozlov, M. Merck's COVID pill loses its lustre: what that means for the pandemic. Nature, doi:10.1038/d41586-021-03667-0 (2021).

16 Prince, T. et al. Antiviral activity of molnupiravir precursor NHC against SARS-CoV-2 Variants of Concern (VOCs) and its therapeutic window in a human lung cell model. bioRxiv, 2021.2011.2023.469695, doi:10.1101/2021.11.23.469695 (2021).

17 Sourimant, J. et al. 4'-Fluorouridine is an oral antiviral that blocks respiratory syncytial virus and SARSCoV-2 replication. Science, eabj5508 (2021).

18 Cox, R. M., Wolf, J. D. \& Plemper, R. K. Therapeutically administered ribonucleoside analogue MK4482/EIDD-2801 blocks SARS-CoV-2 transmission in ferrets. Nat Microbiol 6, 11-18, doi:10.1038/s41564-020-00835-2 (2021).

19 Cox, R. M. et al. Oral prodrug of remdesivir parent GS-441524 is efficacious against SARS-CoV-2 in ferrets. Nat Commun 12, 6415, doi:10.1038/s41467-021-26760-4 (2021).

20 Gruber, A. D., Firsching, T. C., Trimpert, J. \& Dietert, K. Hamster models of COVID-19 pneumonia reviewed: How human can they be? Vet. Pathol., 3009858211057197, doi:10.1177/03009858211057197 (2021).

21 Lee, C. Y. \& Lowen, A. C. Animal models for SARS-CoV-2. Curr. Opin. Virol. 48, 73-81, doi:10.1016/j.coviro.2021.03.009 (2021).

22 Trimpert, J. et al. The Roborovski Dwarf Hamster Is A Highly Susceptible Model for a Rapid and Fatal Course of SARS-CoV-2 Infection. Cell Rep 33, 108488, doi:10.1016/j.celrep.2020.108488 (2020).

23 Zhai, C. et al. Roborovski hamster (Phodopus roborovskii) strain SH101 as a systemic infection model of SARS-CoV-2. Virulence 12, 2430-2442, doi:10.1080/21505594.2021.1972201 (2021).

24 Sheahan, T. P. et al. An orally bioavailable broad-spectrum antiviral inhibits SARS-CoV-2 in human airway epithelial cell cultures and multiple coronaviruses in mice. Sci. Transl. Med. 12, doi:10.1126/scitranslmed.abb5883 (2020). 
25 Painter, W. P. et al. Human Safety, Tolerability, and Pharmacokinetics of Molnupiravir, a Novel BroadSpectrum Oral Antiviral Agent with Activity Against SARS-CoV-2. Antimicrob. Agents Chemother., doi:10.1128/AAC.02428-20 (2021).

26 Oude Munnink, B. B. et al. Transmission of SARS-CoV-2 on mink farms between humans and mink and back to humans. Science 371, 172-177, doi:10.1126/science.abe5901 (2021).

27 Bauer, B. A. \& Besch-Williford, C. in The Laboratory Rabbit, Guinea Pig, Hamster, and Other Rodents (eds M. A. Suckow, K. A. Stevens, \& R. P. Wilson) Ch. 37, 935-946 (Academic Press, 2012).

28 Rosenke, K. et al. Orally delivered MK-4482 inhibits SARS-CoV-2 replication in the Syrian hamster model. Nat Commun 12, 2295, doi:10.1038/s41467-021-22580-8 (2021).

29 Wahl, A. et al. SARS-CoV-2 infection is effectively treated and prevented by EIDD-2801. Nature 591, 451-457, doi:10.1038/s41586-021-03312-w (2021).

30 Fisman, D. N. \& Tuite, A. R. Evaluation of the relative virulence of novel SARS-CoV-2 variants: a retrospective cohort study in Ontario, Canada. CMAJ 193, E1619-E1625, doi:10.1503/cmaj.211248 (2021).

31 Ong, S. W. X. et al. Clinical and virological features of SARS-CoV-2 variants of concern: a retrospective cohort study comparing B.1.1.7 (Alpha), B.1.315 (Beta), and B.1.617.2 (Delta). Clin Infect Dis, doi:10.1093/cid/ciab721 (2021).

32 Sheikh, A. et al. SARS-CoV-2 Delta VOC in Scotland: demographics, risk of hospital admission, and vaccine effectiveness. Lancet 397, 2461-2462, doi:10.1016/S0140-6736(21)01358-1 (2021).

33 Nealon, J. \& Cowling, B. J. Omicron severity: milder but not mild. Lancet, doi:10.1016/S01406736(22)00056-3 (2022).

34 Toots, M. et al. Characterization of orally efficacious influenza drug with high resistance barrier in ferrets and human airway epithelia. Sci. Transl. Med. 11, doi:10.1126/scitranslmed.aax5866 (2019).

35 Urakova, N. et al. beta-d-N (4)-Hydroxycytidine Is a Potent Anti-alphavirus Compound That Induces a High Level of Mutations in the Viral Genome. J. Virol. 92, doi:10.1128/JVI.01965-17 (2018).

36 Agostini, M. L. et al. Small-Molecule Antiviral beta-d-N (4)-Hydroxycytidine Inhibits a ProofreadingIntact Coronavirus with a High Genetic Barrier to Resistance. J. Virol. 93, doi:10.1128/JVI.01348-19 (2019).

37 Yoon, J. J. et al. Orally Efficacious Broad-Spectrum Ribonucleoside Analog Inhibitor of Influenza and Respiratory Syncytial Viruses. Antimicrob. Agents Chemother. 62, doi:10.1128/AAC.00766-18 (2018).

38 Richard, M. et al. SARS-CoV-2 is transmitted via contact and via the air between ferrets. Nat Commun 11, 3496, doi:10.1038/s41467-020-17367-2 (2020).

39 Enserink, M. Coronavirus rips through Dutch mink farms, triggering culls. Science 368, 1169, doi:10.1126/science.368.6496.1169 (2020).

40 Remmer, H. The role of theliver in drug metabolism. Am. J. Med. 49, 617-629, doi:10.1016/s00029343(70)80129-2 (1970). 

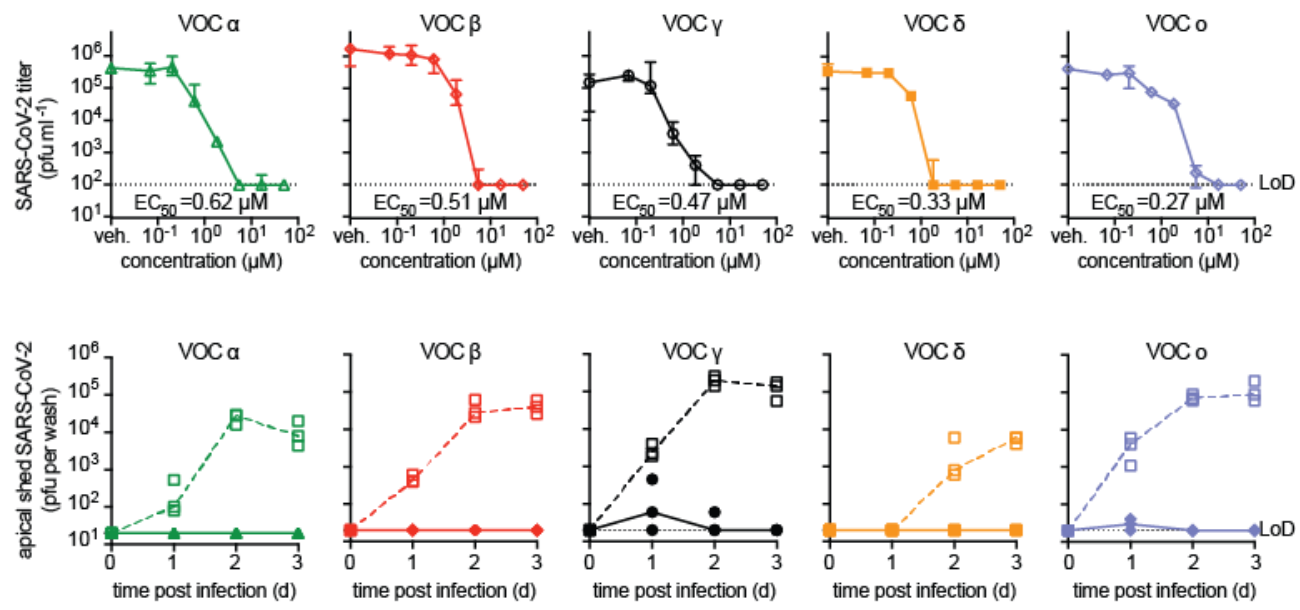

$\Delta \bullet \bullet \bullet \mathrm{NHC} 10 \mu \mathrm{M}$

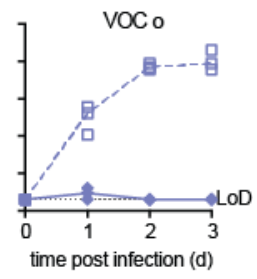

mock
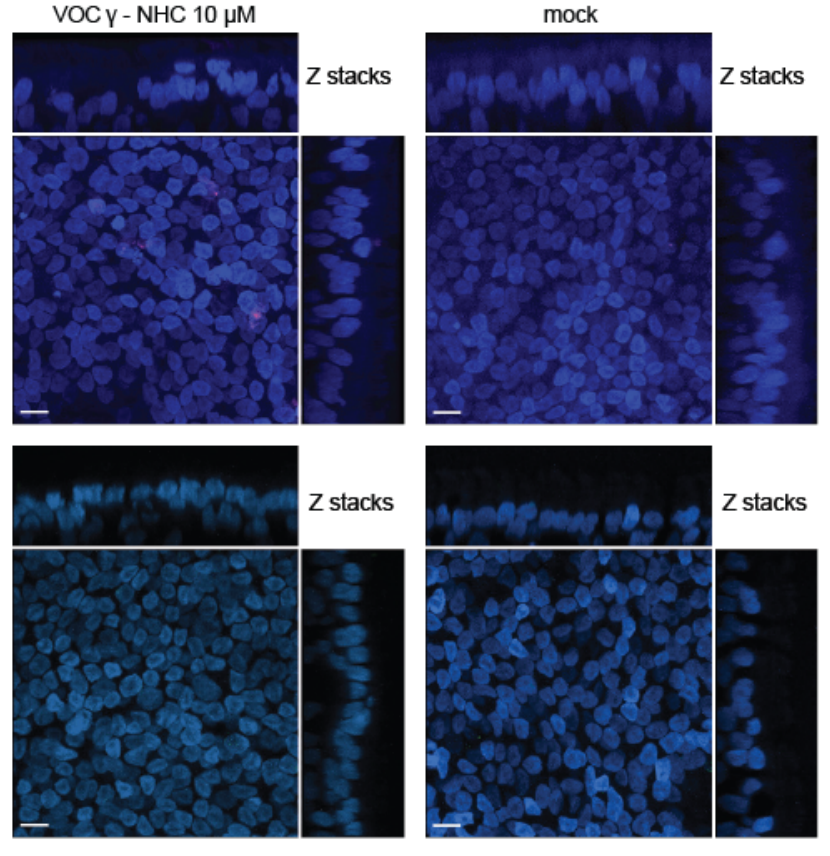
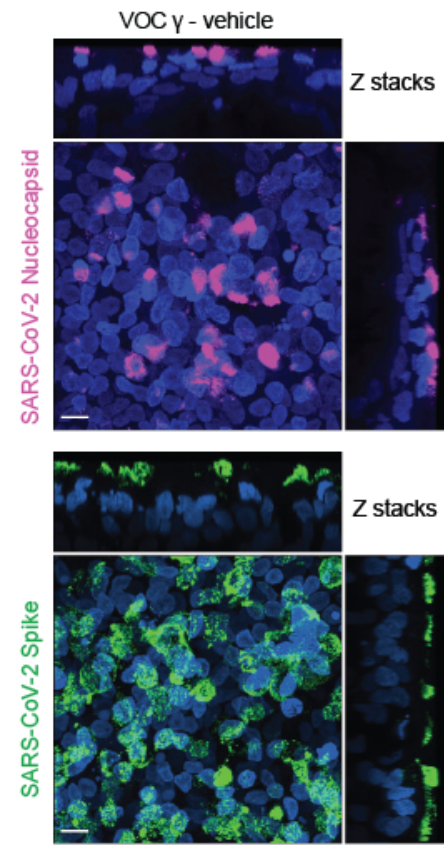

Fig. 1. Efficacy of NHC against VOC in cultured cells and primary HAEs. a, NHC dose-response assays against SARS-CoV-2 VOC alpha, beta, gamma, delta, and omicron on VeroE6 cells. EC 50 values from non-linear

$$
\text { regression modeling are shown. b, Activity of } 10 \mu \mathrm{M} \text { basolateral NHC administered against VOC as in (a) on }
$$

3 well-differentiated primary HAE organoids. Apically shed virus was harvested every 24 hours during a 3-day

4 period after infection. Lines (a-b) intersect group medians $\pm 95 \%$ confidence intervals. LoD, limit of detection. c,

5 Confocal microscopy of primary HAE organoids infected with SARS-CoV-2 VOC gamma. Basolateral NHC is

$$
\text { sterilizing at } 10 \mu \mathrm{M} \text {. SARS-CoV-2 nucleocapsid (pink; } \alpha-S A R S-C o V-2 \text { nucleocapsid), SARS-CoV-2 spike }
$$

7 (green; $\alpha$-SARS-CoV-2 S) and nuclei (DAPI) were detected. Z stacks are shown, scale bars $10 \mu \mathrm{m}$.

8 Representative fields of view are shown. 
bioRxiv preprint doi: https://doi.org/10.1101/2022.02.04.479171; this version posted February 7,2022 . The copyright holder for this preprint (which was not certified by peer review) is the author/funder. All rights reserved. No reuse allowed without permission.

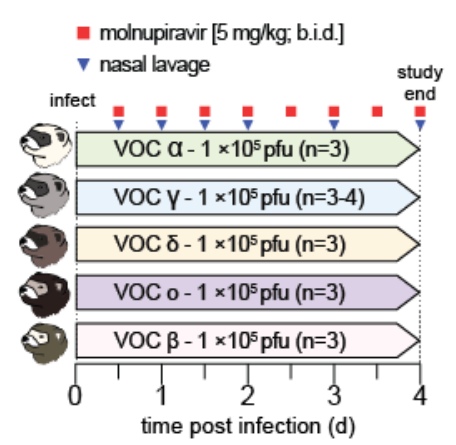

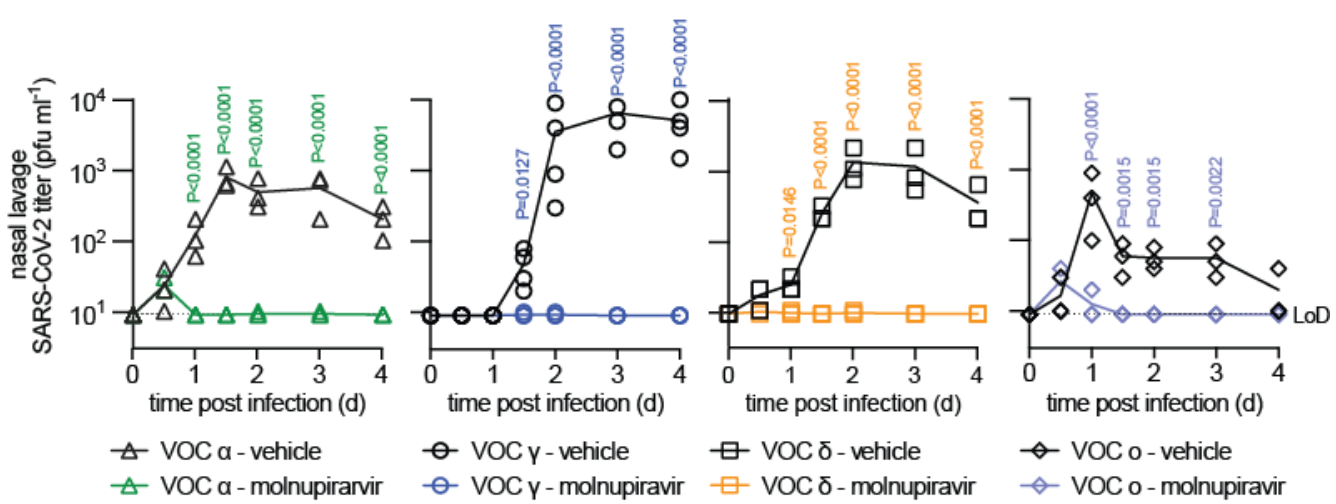

C treat and monitor infected ferrets

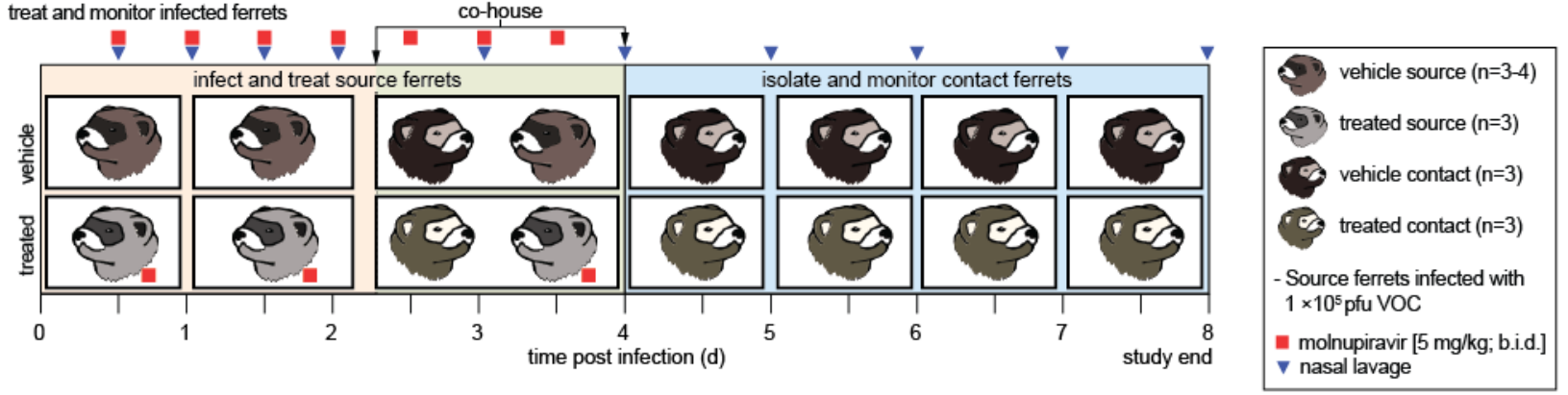

d

$\operatorname{VOc} \alpha$
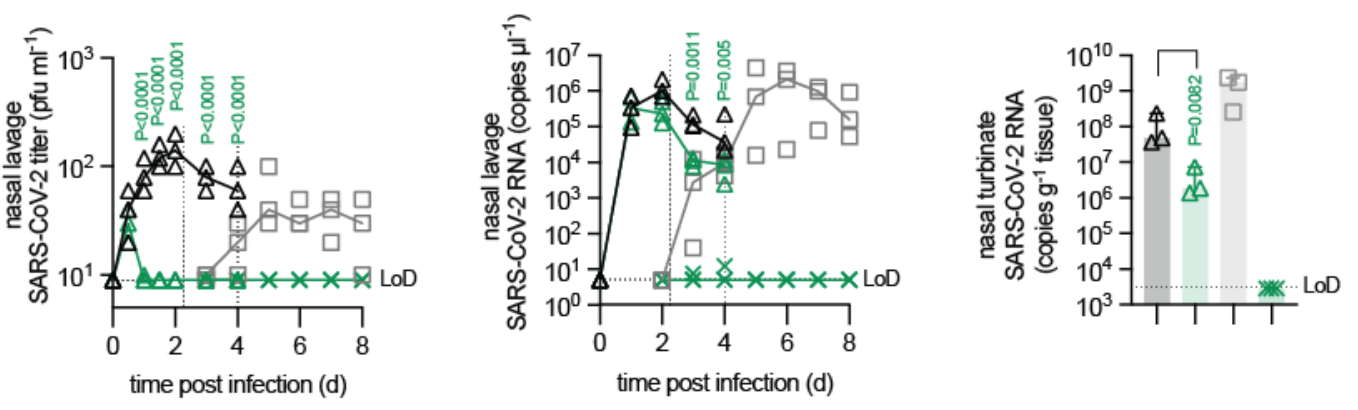

$\triangle \operatorname{VOC} \alpha$ - vehicle $(n=3)$

$\triangle \operatorname{VOC} \alpha$ - molnupiravir $(n=3)$

$\boxminus$ contact - VOC $\alpha$ - vehicle $(n=3)$

$*$ contact - VOC $\alpha$ - molnupiravir $(n=3)$

e

VOC $y$
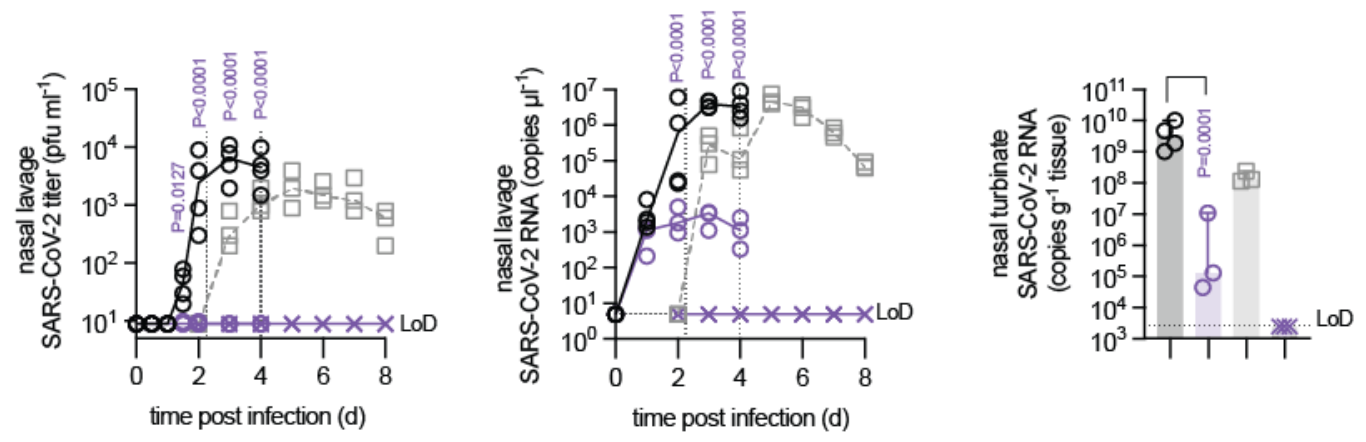

$\vartheta \operatorname{VOC}_{Y}$ - vehicle $(n=4)$

$\ominus$ VOC $Y$ - molnupiravir $(n=3)$

$-\exists$ contact - VOC Y - vehicle $(n=3)$

$*$ contact - VOC $y$ - molnupiravir $(n=3)$

VOC o
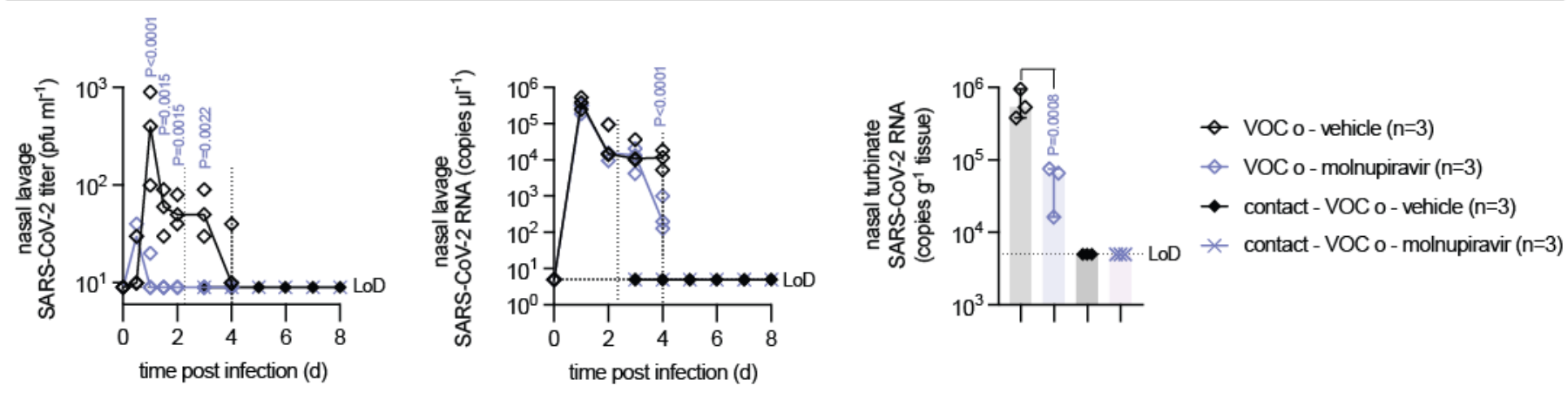
0 Fig. 2. Therapeutic molnupiravir is efficacious against a panel of VOC in ferrets. a, Treatment and

1 monitoring schematic. Ferrets ( $n=6$ total per VOC tested) were infected intranasally with $1 \times 10^{5}$ pfu of VOC and 2 treated orally with vehicle or molnupiravir $(5 \mathrm{mg} / \mathrm{kg}$ b.i.d.), starting 12 hours after infection. b, Infectious SARS3 CoV-2 titers in nasal lavages obtained from vehicle or molnupiravir-treated animals, infected with the specified 4 VOC. c, Schematic of the transmission studies carried out with VOC alpha, gamma, and omicron. For VOC alpha, 5 fresh source ferrets $(n=6)$ were inoculated intranasally with $1 \times 10^{5}$ pfu. For VOC gamma and omicron, 6 transmission arms were added to the infected source ferrets from (b). In all cases, molnupiravir treatment of source 7 ferrets commenced 12 hours after infection at $5 \mathrm{mg} / \mathrm{kg}$ b.i.d. Source animals were co-housed at a 1:1-ratio with 8 uninfected and untreated contact animals starting 42 hours after the beginning of treatment. Nasal lavages were 9 obtained twice daily from source animals in the first 48 hours after infection, thereafter once daily from all animals 0 until termination. Nasal turbinates were extracted from source animals 4 days after infection and untreated 1 sentinels continued in isolation for an additional 4 days. d-f, Transmission study with VOC alpha (d), gamma (e), 2 and omicron (f). Shown for each are infectious titers in nasal lavages (left), corresponding viral RNA copies in 3 the lavages (center), and nasal turbinate titers (right). Symbols in (b, d-f) represent independent biological repeats 4 (virus load of individual animals), lines (b, d-f) intersect group medians, and columns (c, e-g) show group medians $5 \pm 95 \%$ confidence intervals. Statistical analysis with 1-way (d-f turbinate titers) or 2-way (b, d-f lavage titers) 6 ANOVA with Tukey's (d-f turbinate titers) or Sidak's (b, d-f lavage titers) post-hoc multiple comparison tests. P 7 values are shown; LoD, limit of detection. 

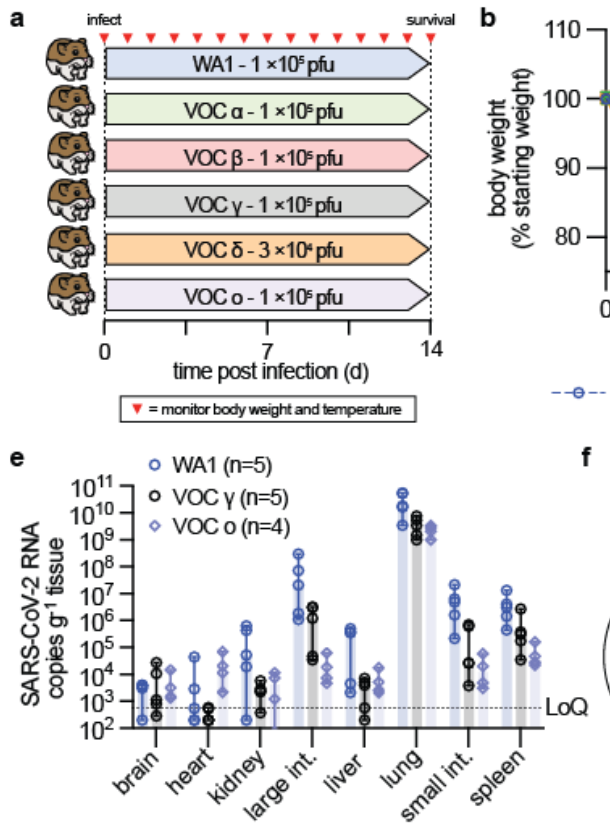

h

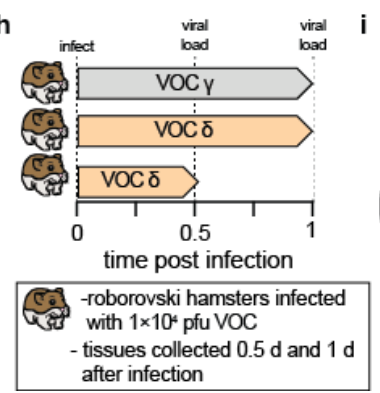

f

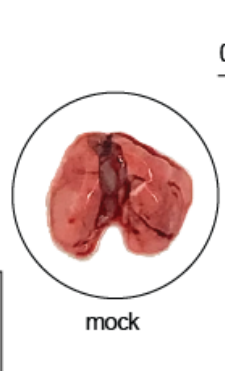

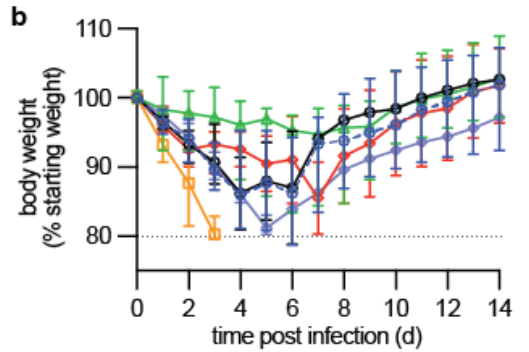

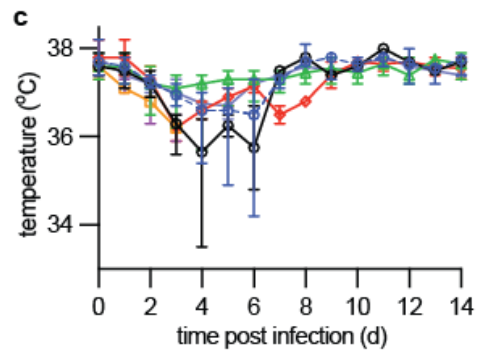

$-\odot--\operatorname{WA1}(n=14) \longrightarrow \operatorname{VOC} \alpha(n=5) \multimap \operatorname{VOC} \beta(n=5) \rightarrow \operatorname{VOC} Y(n=14)$
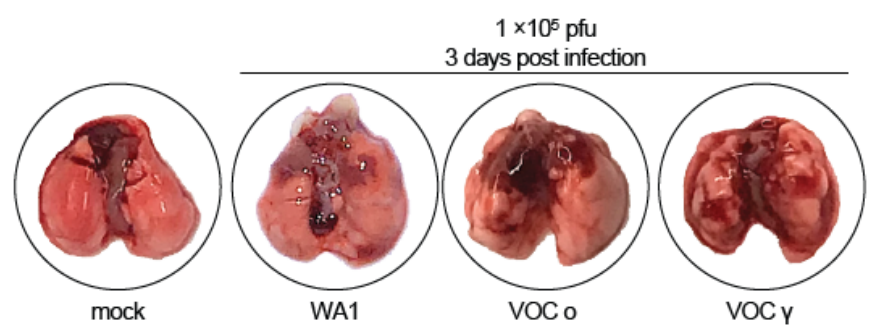

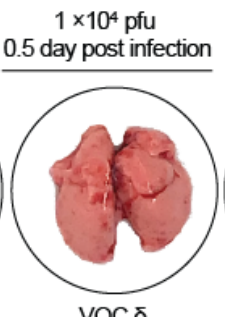

VOC $\bar{~}$

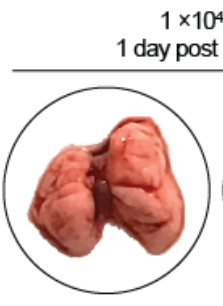

VOC $\overline{0}$ post infection

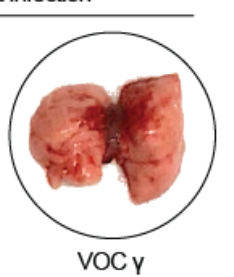

j

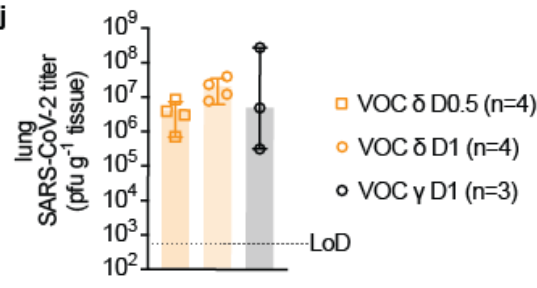

。
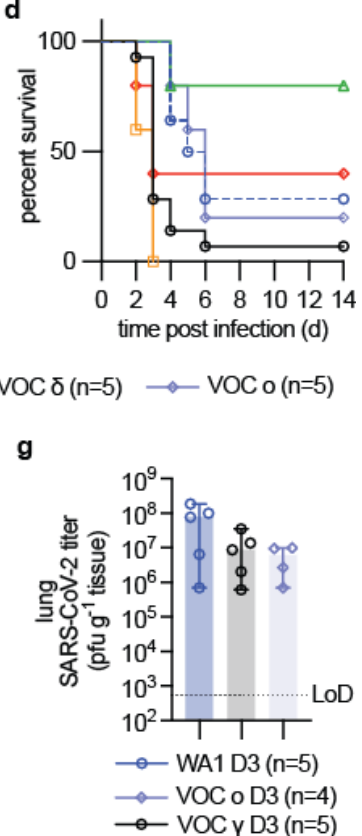

$\operatorname{VOC} \delta(n=5) \multimap \operatorname{VOC} o(n=5)$

Fig. 3. VOC pathogenesis in Roborovski dwarf hamsters. a, Schematic of the dwarf hamster pathogenesis

study. Animals were monitored for up to 14 days after intranasal infection with $1 \times 10^{5}$ pfu each (VOC delta

$3 \times 10^{4}$ pfu per animal). Clinical signs were assessed once daily (red triangles). b-c, Body weight (b) and

temperature (c) of infected dwarf hamsters. d, Survival curves of infected dwarf hamsters from (a). e, Viral RNA

copies in select organs extracted from infected hamsters 3 days after infection. $\mathbf{f}$, Images of lungs from hamsters

mock infected or inoculated with $1 \times 10^{5}$ pfu WA1, VOC omicron, or VOC gamma 3 days after infection. g,

Infectious titers from the lungs of hamsters shown in (f). $\mathbf{h}$, Schematic of the dwarf hamster pathogenesis study

utilizing $1 \times 10^{4} \mathrm{pfu}$ VOC delta and gamma. i, Images of lungs extracted from hamsters mock infected or inoculated with $1 \times 10^{4} \mathrm{pfu}$ VOC omicron or VOC gamma 0.5 (delta) and 1 day (delta and gamma) after infection.

j, Infectious titers from the lungs of hamsters shown in (i). Symbols represent independent biological repeats (e, $\mathrm{g}, \mathrm{j}$ ), lines intersect group medians (b-c), columns show group medians (e, g, j), and error bars represent $95 \%$ 
bioRxiv preprint doi: https://doi.org/10.1101/2022.02.04.479171; this version posted February 7, 2022. The copyright holder for this preprint (which was not certified by peer review) is the author/funder. All rights reserved. No reuse allowed without permission.
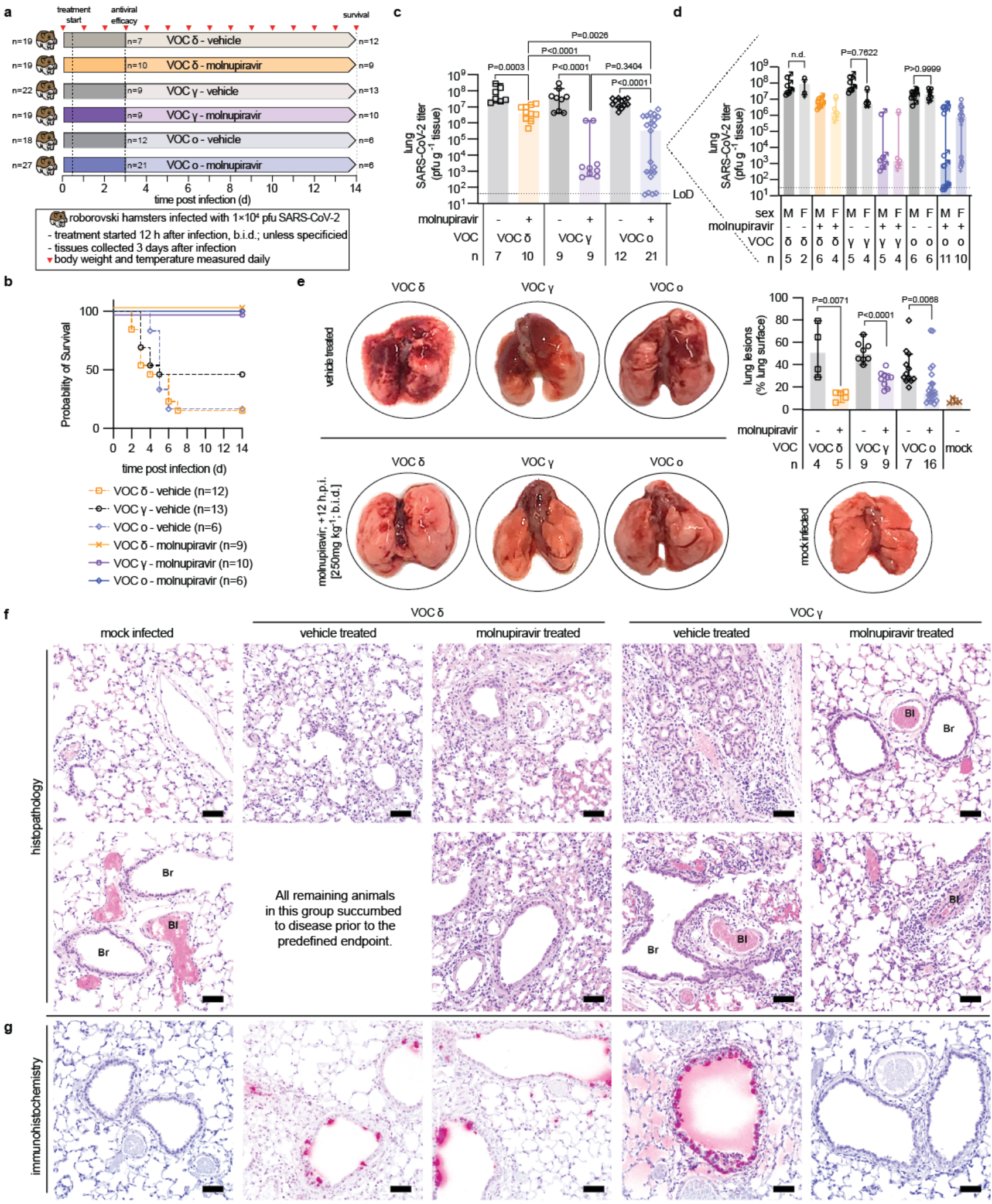

2 Fig 4. Effects of therapeutic molnupiravir on SARS-CoV-2-induced acute lung injury. a, Schematic of the

3 dwarf hamster efficacy study. Animals were monitored for up to 14 days after intranasal infection with $1 \times 10^{4}$

4 pfu each. Clinical signs were assessed once daily (red triangles). Groups of hamsters were euthanized 3 days after

5 infection to assess viral load and perform histological examinations. b, Survival curves of infected dwarf hamsters 
6 from (a). c, Infectious titers from the lungs of hamsters 3 days after infection as shown in (a). d, Role of biological

7 sex on antiviral efficacy shown in (c). e, Images of lungs from hamsters mock infected or inoculated with $1 \times 10^{4}$

8 pfu VOC delta, gamma, or omicron, treated with vehicle or molnupiravir, and harvested 3 days after infection.

9 Quantitation of macroscopic lesions as a percent of total visible lung surface area for infected hamsters treated

0 with vehicle or molnupiravir are shown. Mock infected lungs $(n=4)$ were included as a reference. $\mathbf{f}-\mathbf{g}$,

1 Histopathology (f) and immunohistochemistry (g) of dwarf hamster lung slices from groups harvested 3 days

2 after infection. Staining with hematoxylin and eosin (f) or $\alpha-S A R S-C o V-2 ~ S(g)$. Br, bronchioles; B1, blood

3 vessel; scale bar $50 \mu \mathrm{m}$. Symbols represent independent biological repeats, columns show a group medians (c, d,

4 e), and error bars represent 95\% confidence intervals. Significance was determined using unpaired t-tests (c, d,

5 e) or 2-way (d) ANOVA with Sidak's (d) post-hoc multiple comparison tests; P values are shown.

7 Methods

\section{Study Design}

9 Ferrets (Mustela putorius furo) and Roborovski dwarf hamsters (Phodopus roborovskii) were used as in vivo 0 models to assess the therapeutic efficacy of orally administered molnupiravir against infections with different 1 SARS-CoV-2 VOC. Ferrets were used the examine the effect of molnupiravir on upper respiratory infection and 2 transmission. Roborovski dwarf hamsters were used to study the effects of molnupiravir treatment on severe 3 disease associated with lower respiratory tract infection and acute lung injury. VOC were administered to animals 4 through intranasal inoculation. For ferrets, upper respiratory tract viral titers were assessed routinely through nasal 5 lavages and upper respiratory tract tissues. For hamsters, animals were monitored twice daily for clinical signs 6 and viral loads were determined in respiratory tract tissues at endpoint. Virus loads were determined by plaque 7 assays and RT-qPCR quantitation.

8 Cells

9 African green monkey kidney cells VeroE6 (ATCC CRL-1586 ${ }^{\mathrm{TM}}$ ), Calu-3 (ATCC HB-55 ${ }^{\mathrm{TM}}$ ), and VeroE6$0 \quad$ TMPRSS2 (BPS Bioscience \#78081) were cultivated at $37^{\circ} \mathrm{C}$ with $5 \% \mathrm{CO}_{2}$ in Dulbecco's Modified Eagle's 1 Medium (DMEM) supplemented with 7.5\% heat-inactivated fetal bovine serum (FBS). Normal human 
2 bronchial/tracheal epithelial cells (NHBE) (Lonza Bioscience, cat\# CC-2540S, lot\# 0000646466, passage 3, 3 donor "M4") from a 38-year-old male were expanded in PneumaCult-Ex Plus (Stemcell Technologies cat\# 05040) 4 and differentiated in PneumaCult-ALI (Stemcell Technologies cat\# 05001) for 8 weeks in following the 5 manufacturer's instructions. All cells were authenticated and checked for mycoplasma prior to use.

\section{Antibodies}

7 SARS-CoV-2 $\mathrm{N}$ and goblet cells were co-stained using rabbit anti-SARS-CoV-2 Nucleocapsid monoclonal 8 antibody (HL453) (Invitrogen, cat\# MA5-36272) (1:100 dilution) and mouse anti-MUC5AC (ThermoFisher, cat\# 9 MA5-12175) (1:200 dilution) as primary antibodies, respectively. Donkey anti-goat (Alexa Fluor ${ }^{\circledR} 568$ 0 (ThermoFisher Scientific, cat\# A-11057)) and rabbit anti-mouse IgG $(\mathrm{H}+\mathrm{L})$ cross-adsorbed secondary antibody 1 (Alexa Fluor ${ }^{\circledR} 488$ (ThermoFisher Scientific, cat\# A-11059)) were used at a 1:500 dilution as secondary 2 antibodies. For staining of SARS-CoV-2 S, mouse anti SARS-CoV-1 and 2 Spike protein clone [1A9] monoclonal 3 (Abcam, cat\# ab273433) (1:200 dilution) and goat anti-mouse IgG (H+L) highly cross-adsorbed secondary 4 antibody (Alexa Fluor ${ }^{\circledR}$ 488; 1:500 dilution (Invitrogen, cat\# A-11029)), were used as primary and secondary 5 antibodies, respectively. For staining of ciliated cells, rabbit anti-beta IV tubulin recombinant antibody conjugated 6 with Alexa Fluor ${ }^{\circledR} 647$ [EPR16775] (Abcam, cat\# ab204034) was used at a 1:100 dilution.

\section{$7 \quad$ Viruses}

8 The following SARS-CoV-2 isolates were obtained from BEI resources, amplified on Calu-3 cells. SARS-CoV92 (WA1; lineage A, isolate USA-WA1/2020, BEI cat\# NR-52281), VOC alpha (lineage B.1.1.7, isolate 0 USA/CA_CDC_5574/2020, BEI cat\# NR-54011), VOC beta (lineage B.1.351, isolate hCoV-19/South 1 Africa/KRISP-K005325/2020, BEI cat\# NR-54009), and VOC gamma (lineage P.1., isolate hCoV2 19/Japan/TY7-503/2021 (Brazil P.1), BEI cat\# NR-54982). VOC delta (lineage B.1.617.2, clinical isolate 3 \#2333067) and VOC omicron (lineage B.1.1.529, WA-UW-21120120771) were obtained from the Northwestern

4 Reference laboratory and amplified on Calu-3 cells. All viruses were authenticated by whole genome next 5 generation sequencing prior to use. 
$7 \quad 12$-well plates were seeded with $2 \times 10^{5}$ cells per well the day before infection. Each isolate was diluted in DMEM

8 to achieve a multiplicity of infection (MOI) of $0.1 \mathrm{pfu} / \mathrm{cell}$, adsorbed on cells for 1 hour at $37^{\circ} \mathrm{C}$ following which

9 the inoculum was removed and replaced with DMEM with 2\% heat-inactivated FBS. The media contained

0 additionally $0.1 \%$ DMSO (vehicle) and the indicated concentration of NHC (EIDD-1931). After 48 hours at

$137^{\circ} \mathrm{C}$, the cell supernatant was harvested aliquoted and frozen at $-80^{\circ} \mathrm{C}$ before titration by standard plaque assay.

2 Log viral titers were normalized using the average top plateau of viral titers to define $100 \%$ and were analyzed

3 with a non-linear regression with variable slope to determine $\mathrm{EC}_{50}$ (Prism; GraphPad).

\section{Confocal microscopy}

5 Eight-week differentiated HAE cells were infected (or mock-infected) with $3 \times 10^{4}$ pfu of SARS-CoV-2 VOC 6 and fixed at day 3 after infection. For co-staining of SARS-CoV-2 N and goblet cells, cells were permeabilized 7 for 30 minutes with a $200 \mu \mathrm{l}$ apical overlay of PBS with $0.1 \%$ Triton X-100 and 3\% bovine serum albumin (BSA), 8 then the overlay was removed and cells were apically incubated with $100 \mu$ of primary antibodies in wash buffer 9 (PBS with $0.05 \%$ Tween20 and $0.3 \%$ BSA) for 1 hour at RT. After three 5-minute $200 \mu 1$-washes with wash 0 buffer, cells were incubated with secondary antibodies in wash buffer for 1 hour at RT. Cells were subsequently 1 incubated with Hoechst 34580 (1:1000 dilution in wash buffer) for 5 minutes and washed three times. Membranes 2 were then excised and mounted using Prolong Diamond antifade mountant overnight at RT (ThermoFisher 3 Scientific). For co-staining of SARS-CoV-2 S and ciliated cells, the same method was used with a saturation step 4 with PBS with $0.05 \%$ Tween 20 and 3\% BSA in lieu of a permeabilization step. Image captures were performed 5 with a Zeiss Axio Observer Z.1 and an LSM 800 confocal microscope with AiryScan, controlled with the Zeiss

6 Zen 3.1 Blue software package (Windows 10). Representative pictures were taken either with a $63 \times$ Plan7 Apochromat (NA: 1.40, oil) objective. Digital pictures are pseudocolored for optimal presentation. For clarity of 8 presentation, the intensity of staining was digitally balanced in between each different virus isolates with Zeiss 9 Zen Blue software, while keeping the same processing for the conditions that are directly compared (vehicle vs 0 treatment).

$1 \quad$ Plaque assay 
2 Samples were serially diluted (tenfold dilutions starting at a 1:10 initial dilution) in DMEM medium supplemented

3 with 2\% FBS containing Antibiotic-Antimycotic (Gibco). The serial dilutions were added to Vero E6 cells seeded

4 in 12 -well plates at $3 \times 10^{5}$ cells per well 24 hours previously. The virus was allowed to adsorb for 1 hour at $37^{\circ} \mathrm{C}$.

5 Subsequently, the inoculum was removed, and the cells were overlaid with 1.2\% Avicel (FMC BioPolymer) in

6 DMEM and incubated for 3 days at $37^{\circ} \mathrm{C}$ with $5 \% \mathrm{CO}_{2}$. After three days, the Avicel was removed, cells were

7 washed once with PBS, fixed with $10 \%$ neutral buffered formalin and plaques were visualized using $1 \%$ crystal

8 violet. For hamsters infected with VOC gamma, delta, and omicron, plaque assays were performed with VeroE6-

9 TMPRSS2 cells.

$0 \quad$ In vivo efficacy of molnupiravir in ferrets against VOC

1 Groups of ferrets were inoculated with $1 \times 10^{5} \mathrm{pfu}$ of VOC alpha or delta $(1 \mathrm{ml} ; 0.5 \mathrm{ml}$ per nare). Twelve hours

2 after infection, groups of ferrets were treated twice daily (b.i.d.) with vehicle (1\% methylcellulose) or

3 molnupiravir at a dose of $5 \mathrm{mg} / \mathrm{kg}$ in $1 \%$ methylcellulose. Treatments were administered by oral gavage and

4 continued every 12 hours until 4 days after infection. All ferrets were euthanized 4 days after infection and tissues

5 were harvested to determine SARS-CoV-2 titers and the presence of viral RNA.

$6 \quad$ Efficacy and contact transmission of VOC in ferrets

7 Two groups of six source ferrets were inoculated intranasally with $1 \times 10^{5}$ pfu of VOC alpha or gamma. After 8 twelve hours, groups of source ferrets were further divided into two groups, receiving vehicle ( $\mathrm{n}=3$ for VOC 9 alpha; $\mathrm{n}=4$ for VOC gamma) or molnupiravir treatment $(5 \mathrm{mg} / \mathrm{kg}$ b.i.d.) administered by oral gavage. At 54 hours 0 after infection, each source ferret was co-housed with two uninfected and untreated contact ferrets. Ferrets were 1 co-housed until 96 hours after infection, when sourced ferrets were euthanized and contact ferrets were housed 2 individually. All contact ferrets were monitored for 4 days after separation from source ferrets and then 3 euthanized. Nasal lavages were performed on source ferrets twice daily until cohousing started. After cohousing 4 started, nasal lavages were performed on all ferrets every 24 hours. Nasal turbinates were harvested for all ferrets 5 after euthanization to determine infectious titers and the presence of viral RNA. 
7 Female and male hamsters (2-4 months of age) were purchased from Ron Van Der Vliet, Netherlands. The

8 hamsters were permanently quarantine housed under ABSL-2 conditions until study start. After a minimal resting

9 period of 2 weeks after arrival, animals were randomly assigned to groups for individual studies, transferred into

0 an ABSL-3 facility immediately prior to study start, and housed singly in ventilated negative-pressure cages

1 during the studies. To establish a pathogenicity profile, hamsters were inoculated intranasally with $1 \times 10^{5}$ pfu in

$250 \mu \mathrm{l}\left(25 \mu \mathrm{l}\right.$ per nare), unless otherwise stated (VOC delta $\left.3 \times 10^{4}\right)$. The hamsters were anaesthetized with

3 dexmedetomidine/ketamine before inoculation. Groups of hamsters were euthanized 14 days after infection and

4 their organs were harvested to determine the presence of viral RNA in different tissues (WA1 and VOC gamma

5 only).

\section{' $\quad$ In vivo efficacy of molnupiravir in hamsters}

7 Groups of hamsters were inoculated with $1 \times 10^{4} \mathrm{pfu}$ in $50 \mu \mathrm{l}(25 \mu 1$ per nare $)$. At 12 hours after infection, the 8 hamsters were treated b.i.d. with vehicle (1\% methylcellulose) or molnupiravir at a dosage of $250 \mathrm{mg} / \mathrm{kg}$ body 9 weight, respectively. The compound was administered via oral gavage in $1 \%$ methylcellulose. After the start of 0 treatment, b.i.d. dosing was continued until 12 days after infection. Subgroups of hamsters were euthanized 3 1 days after infection. All studies were terminated 14 days after infection. Organs were harvested to determine virus 2 titers and presence of viral RNA in different tissues.

\section{Titration of SARS-CoV-2 in tissue extracts}

4 For virus titration, the organs were weighed and homogenized in PBS. The homogenates were centrifuged at

2,000g for 5 minutes at $4{ }^{\circ} \mathrm{C}$. The clarified supernatants were harvested, frozen and used in subsequent plaque assays. For detection of viral RNA, the harvested organs were stored in RNAlater at $-80^{\circ} \mathrm{C}$. The tissues were homogenized, and the total RNA was extracted using a RNeasy mini kit (Qiagen).

\section{Quantitation of SARS-CoV-2 RNA copy numbers}

SARS-CoV-2 RNA was detected using the nCoV_IP2 primer-probe set (National Reference Center for

Respiratory Viruses, Institute Pasteur) which targets the SARS-CoV-2 RdRP gene. RT-qPCR reactions were performed using an Applied Biosystems 7500 real-time PCR system using the StepOnePlus real-time PCR 
3 (Thermo Fisher Scientific) to detect viral RNA. A standard curve was created using a PCR fragment (nt 12669-

414146 of the SARS-CoV-2 genome) generated from viral complementary DNA using the nCoV_IP2 forward

5 primer and the $\mathrm{nCoV}_{-}$IP4 reverse primer to quantitate the RNA copy numbers. RNA copy numbers were

6 normalized to the weight of tissues used.

\section{$7 \quad$ Statistics and reproducibility}

8 The Microsoft Excel (versions 16.52) and Numbers (version 10.1) software packages were used for most data

9 collection. The GraphPad Prism (version 9.1.0) software package was used for data analysis. Reverse transcription

$0 \quad$ RT-qPCR data were collected and analyzed using the StepOnePlus (version 2.1; Applied Biosystems) software

1 package. Figures were assembled using Adobe Illustrator (version CS6). T-tests were used to evaluate statistical

2 significance between experiments with two sets of data. One- and two-way ANOVAs with Dunnett's, Tukey’s,

3 or Sidak's comparisons post-hoc tests without further modifications were used to evaluate statistical significance

4 when more than two groups were compared or datasets contained two independent variables, respectively.

5 Specific statistical tests are specified in the figure legends for individual studies. Supplementary Datasets 4,5

6 summarize all quantitative measurements and statistical analyses (effect sized, P values, and degrees of freedom),

7 respectively. Effect sizes between groups were calculated as $\eta^{2}=\left(\mathrm{SS}_{\text {effect }}\right) /\left(\mathrm{SS}_{\text {total }}\right)$ for one-way $\mathrm{ANOVA}$ and $\omega^{2}=$

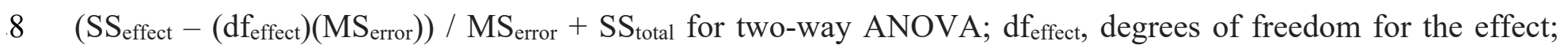

$9 \mathrm{SS}_{\text {total }}$, sum of squares for total; $\mathrm{SS}_{\text {effect, }}$ sum of squares for the effect; $\mathrm{MS}_{\text {error, }}$ mean square error. Effective

0 concentrations for antiviral potency were calculated from dose-response datasets through four-parameter variable

1 slope regression modelling. A biological repeat refers to measurements taken from distinct samples, and the

2 results obtained for each individual biological repeat are shown in the figures along with the exact size (n, number)

3 of biologically independent samples, animals, or independent experiments. The measure of the center (connecting

4 lines and columns) is the mean throughout, unless otherwise specified. The statistical significance level ( $\alpha)$ was

5 set to $<0.05$ for all experiments. Exact $\mathrm{P}$ values are shown in Supplementary Dataset 5 and in individual graphs

6 when possible.

7 Ethics statement 
8 All animal studies were performed in compliance with the Guide for the Care and Use of Laboratory Animals of

9 the National Institutes of Health and the Animal Welfare Act Code of Federal Regulations. Experiments with

0 SARS-CoV-2 involving ferrets and dwarf hamsters were approved by the Georgia State Institutional Animal Care

1 and Use Committee under protocol A20031 and A21019, respectively. All experiments involving infectious

2 SARS-CoV-2 were approved by the Georgia State Institutional Biosafety Committee under protocol B20016 and 3 performed in BSL-3/ABSL-3 facilities at the Georgia State University.

\section{Acknowledgements}

We thank members of the GSU High Containment Core and the Department for Animal Resources for

support, and A. L. Hammond for critical reading of the manuscript. This work was supported, in part, by Public

Health Service grants AI071002 (to RKP) and AI141222 (to RKP), from the NIH/NIAID. The funders had no

role in study design, data collection and interpretation, or the decision to submit the work for publication.

\section{Author Contributions}

2 C.M.L., R.M.C., and R.K.P. conceived and designed the experiments. C.M.L., R.M.C., J.S., J.D.W., and

3 R.K.P. conducted most of the experiments. K.J, Q.P., and A.L.G. performed next-generation sequencing.

4 M.T.S. performed chemical synthesis. M.G.N. and G.R.P. provided critical materials. K.S. performed

5 histopathology of organ samples. C.M.L., R.M.C., J.S., A.L.G., and R.K.P. analyzed the data. R.M.C. and

$6 \quad$ R.K.P. wrote the manuscript.

\section{Competing Interests Declaration}

M.G.N. and G.R.P. are coinventors on patent 20190022116, "N4-Hydroxycytidine and Derivatives and

Anti-Viral Uses Related Thereto", covering composition of matter and method of use of EIDD-2801 for antiviral therapy. This study could affect their personal financial status. All other authors declare no competing interests. 


\section{Additional information}

\section{Supplementary Information}

6 Supplementary Information is available for this paper.

\section{Correspondence}

9 Correspondence and requests for materials should be addressed to Richard K Plemper.

\section{Data Availability}

All data generated and analyzed for this study are included in this published article and its supplementary

3 information files. Quantitative source data for Fig. 1-4, Extended Data 1-8, and Supplementary Fig. 3 are

4 provided with the paper in Supplementary Dataset 4.

\section{Code Availability}

This study does not use custom codes. All commercial computer codes and algorithms used are specified in 8 the Methods section.

\section{Extended Data Legends}

a

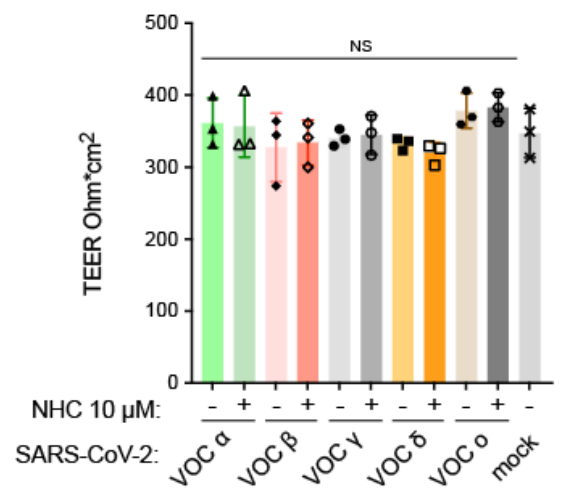

b

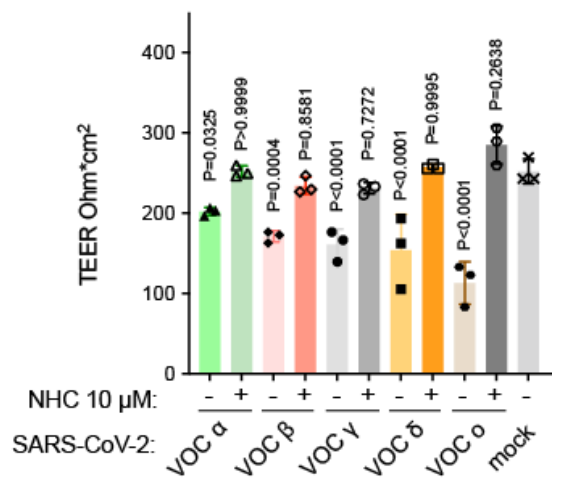

2 Extended Data 1. Transepithelial electrical resistance measurements (TEER). HAE cultures were mock infected

3 ( $\times$ symbols) or infected with VOC alpha (triangles), beta (diamonds), gamma (circles), delta (squares), or omicron 4 (hexagons) in the presence of $10 \mu \mathrm{M}$ NHC (open symbols) or vehicle (0.1\% DMSO; closed symbols). a, TEER 
5 measurements of HAE cultures taken at the time of infection with different SARS-CoV-2 VOC. b, TEER

6 measurements of HAE cultures taken 3 days after infection with different SARS-CoV-2 VOC. Symbols represent

7 independent biological repeats. P values are shown. 1-way ANOVA with Dunnett's post hoc multiple comparison

8 tests (a-b). Bar graphs show the group mean \pm SD.

9

a

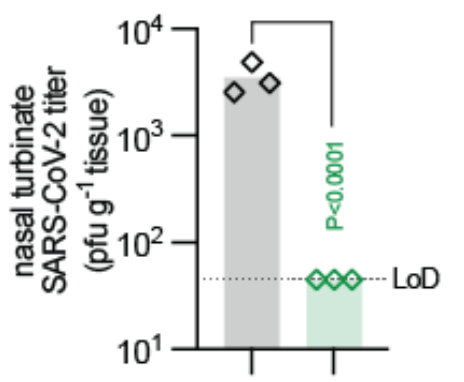

$\diamond$ VOC $\delta$ - vehicle $(\mathrm{n}=3)$

$\diamond \operatorname{VOC} \delta$ - molnupiravir $(n=3)$

b

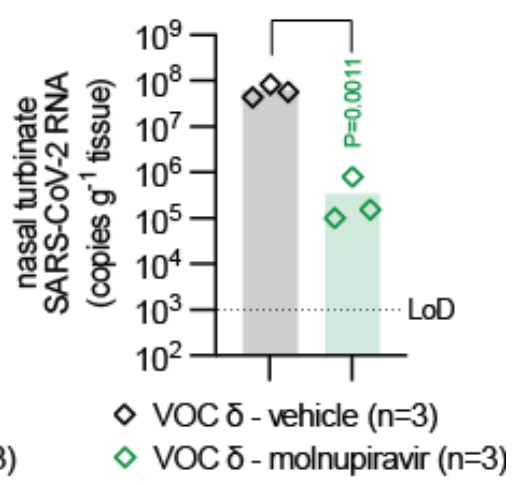

2 Extended Data 2. Viral RNA and upper respiratory tract viral titers from VOC infected ferrets. a, Infectious

3 titers of SARS-CoV-2 in nasal turbinates harvested 4 days after infection. b, SARS-CoV-2 RNA copies present

4 in nasal lavages from ferrets infected with VOC delta and treated with vehicle or molnupiravir. LoD limit of

5 detection. Two-tailed t-test (a-b); P values are shown. Symbols represent independent biological repeats

6 (individual animals). Bar graphs show the group mean.

a

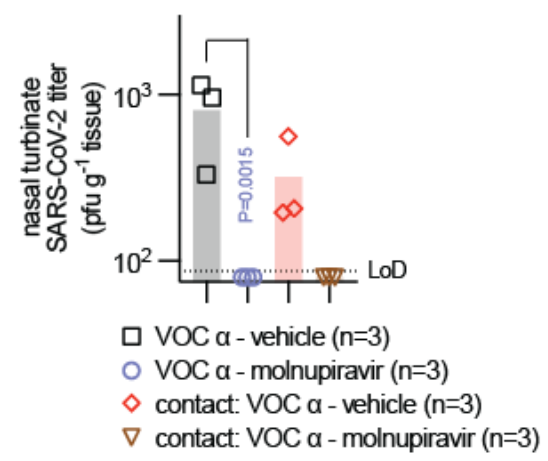

b

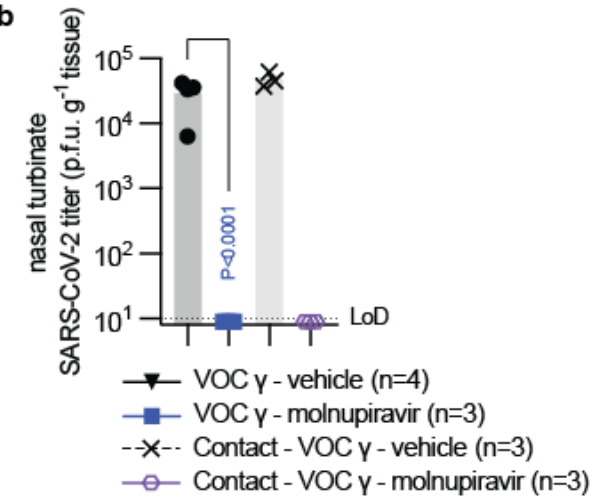

c

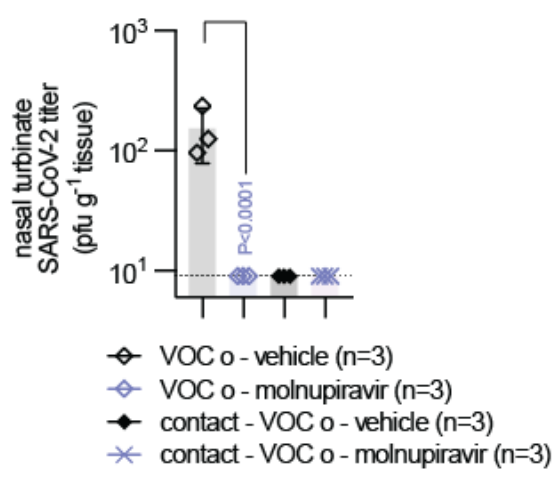

9 Extended Data 3. Viral RNA and upper respiratory tract viral titers from VOC infected ferrets. a, Infectious 0 titers of SARS-CoV-2 VOC alpha in nasal turbinates harvested 4 days after infection of source ferrets treated 
1 with vehicle (black squares) or molnupiravir (blue circles) and of untreated vehicle (red diamonds) or

2 molnupiravir (brown triangles) contact ferrets. b, Infectious titers of SARS-CoV-2 VOC gamma in nasal

3 turbinates harvested 4 days after infection of source ferrets treated with vehicle (black triangles) or molnupiravir

4 (blue squares) and of untreated vehicle (brown $\times$ symbols) or molnupiravir (purple hexagons) contact ferrets. c,

5 Infectious titers of SARS-CoV-2 VOC gamma in nasal turbinates harvested 4 days after infection of source

6 ferrets treated with vehicle (black diamonds) or molnupiravir (blue diamonds) and of untreated vehicle (black

7 diamonds) or molnupiravir (blue $\times$ symbols) contact ferrets. LoD limit of detection. 1-way ANOVA with

8 Tukey's (a-c) post hoc multiple comparison tests; P values are shown. Symbols represent independent

9 biological repeats (individual animals). Bar graphs show the group mean. Error bars represent \pm SD.

0

\begin{tabular}{|c|c|c|c|c|c|}
\hline ID & $\begin{array}{c}\text { median } \\
\text { survival (d) }\end{array}$ & survival (\%) & $\begin{array}{c}\text { animal } \\
\text { number (n) }\end{array}$ & comparison & $P$ value \\
\hline \multirow[t]{6}{*}{ WA1 } & 5.75 & 28.571 & 14 & -- & n.a. \\
\hline & & & & $\operatorname{VOC} \alpha$ & 0.0873 \\
\hline & & & & VOC $\beta$ & 0.6119 \\
\hline & & & & $\operatorname{VOC} \gamma$ & 0.0031 \\
\hline & & & & VOC $\delta$ & $<0.0001$ \\
\hline & & & & VOC o & 0.9988 \\
\hline \multirow[t]{5}{*}{$\operatorname{VOC} \alpha$} & n.d. & 80 & 5 & -- & n.a. \\
\hline & & & & VOC $\beta$ & 0.1591 \\
\hline & & & & $\operatorname{VOC} \gamma$ & 0.0032 \\
\hline & & & & VOC $\delta$ & 0.0031 \\
\hline & & & & VOC o & 0.1177 \\
\hline \multirow[t]{4}{*}{ VOC $\beta$} & 3 & 40 & 5 & -- & n.a. \\
\hline & & & & $\mathrm{VOC} \gamma$ & 0.3278 \\
\hline & & & & VOC $\delta$ & 0.1729 \\
\hline & & & & VOC o & 0.8741 \\
\hline \multirow[t]{3}{*}{$\mathrm{VOC} \gamma$} & 3 & 7.143 & 14 & -- & n.a. \\
\hline & & & & VOC $\delta$ & 0.0505 \\
\hline & & & & VOC o & 0.0555 \\
\hline \multirow[t]{2}{*}{ VOC $\delta$} & 3 & 0 & 5 & -- & n.a. \\
\hline & & & & VOC o & 0.0031 \\
\hline VOC o & 6 & 20 & 5 & -- & n.a. \\
\hline
\end{tabular}

1 Extended Data 4. Analysis of dwarf hamster survival after infection with different VOC. Significance was

2 determined with Mantel-Cox test. 
a

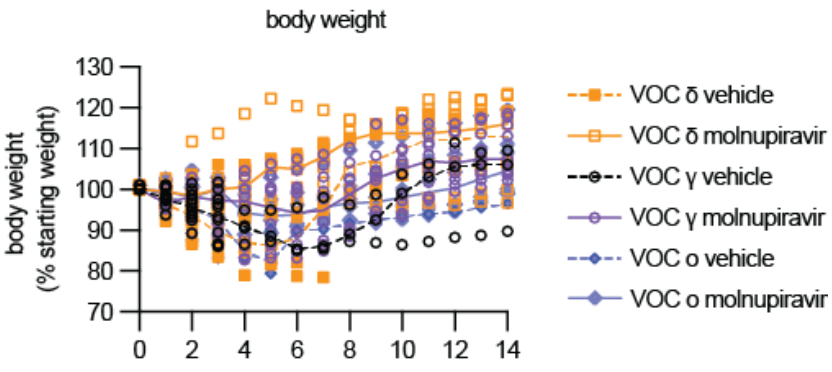

b

temperature

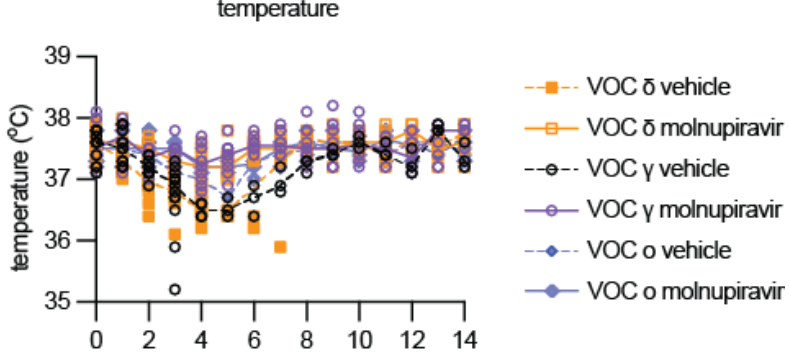

5 Extended Data 5. Clinical signs during treatment with molnupiravir. Roborovski dwarf hamsters infected with

$61 \times 10^{4} \mathrm{pfu}$ VOC gamma, VOC delta, or VOC omicron and treated with molnupiravir or vehicle were infected 7 and monitored for clinical signs for 14 days. a-b, Body weight (a) and temperature were measured once daily.

8 Symbols represent independent biological repeats (individual animals).

a

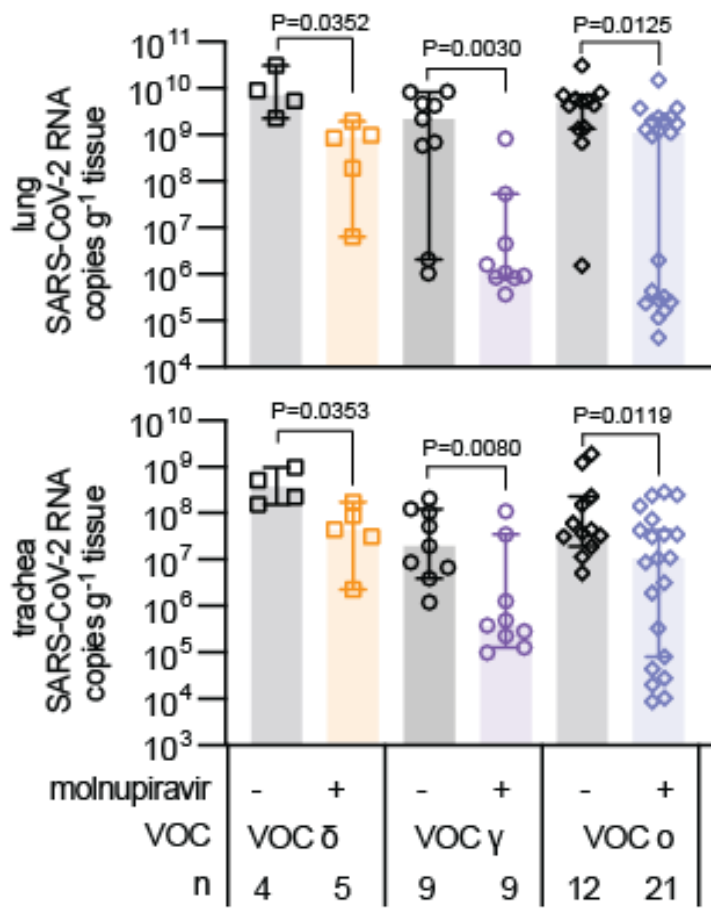


1 Extended Data 6. SARS-CoV-2 RNA copies in hamsters infected with different VOC. RNA copies were

2 determined in hamsters infected with $1 \times 10^{4} \mathrm{pfu}$ VOC gamma, VOC delta, or VOC omicron treated with

3 molnupiravir or vehicle. a, SARS-CoV-2 RNA copies present in lungs (top) and trachea (bottom) of infected

4 hamsters. Symbols represent independent biological repeats (n, individual animals), columns show group

5 medians and error bars represent the $95 \%$ confidence intervals. Significance was determined using unpaired t-

6 tests; P values are shown.

a vehicle treated survival
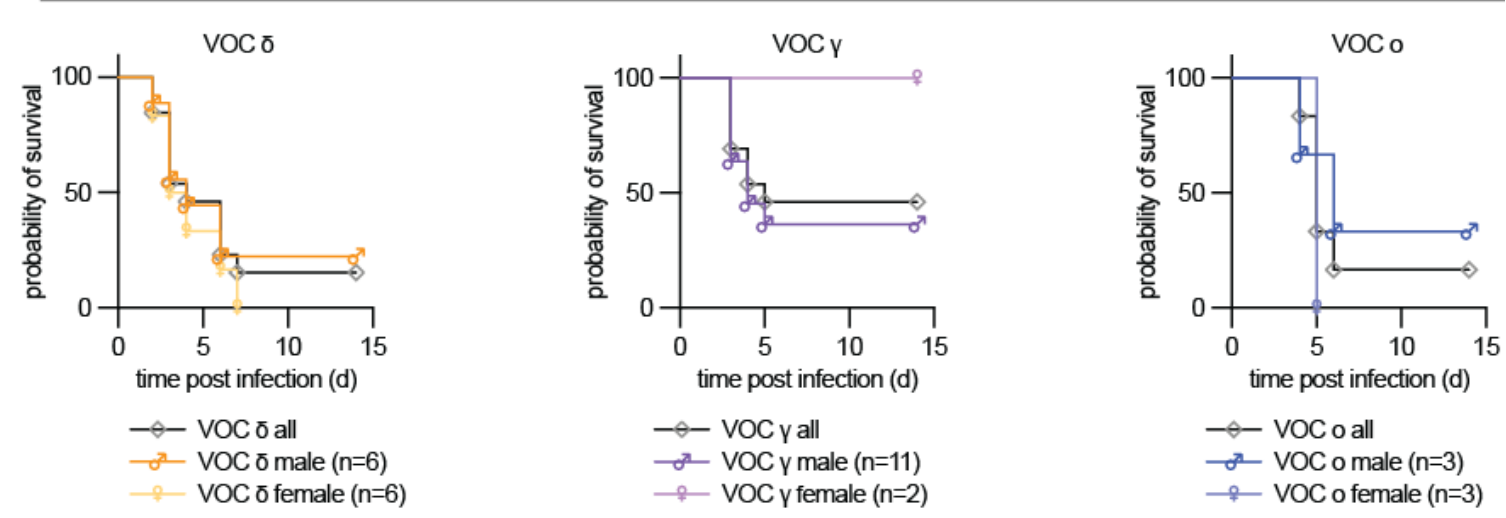

9 Extended Data 7. Effect of biological sex on survival of vehicle treated hamsters infected with $1 \times 10^{4} \mathrm{pfu}$ of

0 VOC gamma, VOC delta, or VOC omicron. Survival curves for females ( $Q$ symbol) and males (ð symbol) are 1 shown.

\begin{tabular}{|c|c|c|c|c|}
\hline \multirow{6}{*}{ 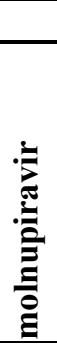 } & \multirow{6}{*}{$\begin{array}{c}\text { all } \\
\text { male } \\
\text { female }\end{array}$} & \multicolumn{3}{|c|}{ VOC delta } \\
\hline & & \multicolumn{3}{|c|}{ molnupiravir } \\
\hline & & all & male & female \\
\hline & & n.a. & 0.9992 & 0.987 \\
\hline & & 0.9992 & n.a. & 0.7337 \\
\hline & & 0.987 & 0.7337 & n.a. \\
\hline \multirow{3}{*}{$\begin{array}{l}\frac{0}{\varrho} \\
\frac{\pi}{0}\end{array}$} & all & 0.0003 & 0.0126 & 0.0003 \\
\hline & male & 0.0011 & 0.0272 & 0.0007 \\
\hline & female & n.d. & n.d. & n.d. \\
\hline & & \multicolumn{3}{|c|}{ VOC gamma } \\
\hline \multirow[b]{2}{*}{$\overline{\mathrm{e}}$} & & \multicolumn{3}{|c|}{ molnupiravir } \\
\hline & all & n.a. & 0.9999 & 0.9999 \\
\hline
\end{tabular}




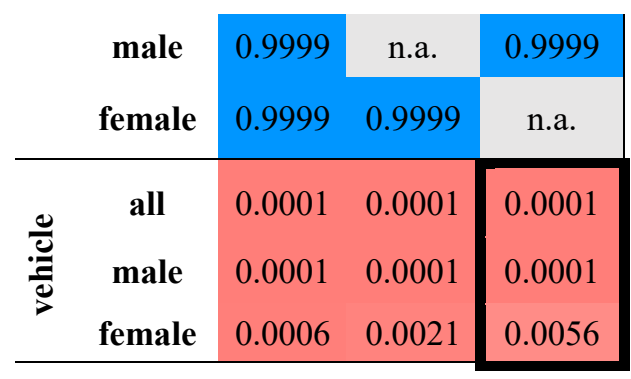

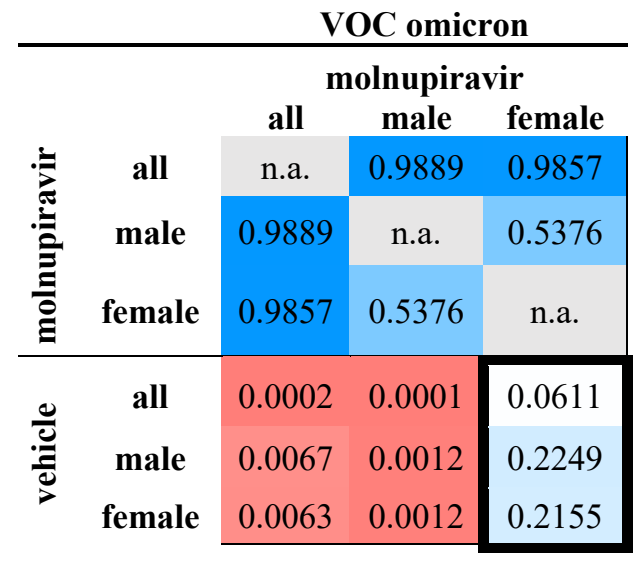

4 Extended Data 8. Analysis of biological sex as a variable in molnupiravir efficacy in dwarf hamsters.

5 Significance was determined using 2-way ANOVA with Sidak's multiple comparison post-hoc test. Numbers 6 specify $\mathrm{P}$ values; blue shading, $\mathrm{P}$ values $>0.05$; red shading, $\mathrm{P}$ values $<0.05$. 
bioRxiv preprint doi: https://doi.org/10.1101/2022.02.04.479171; this version posted February 7, 2022. The copyright holder for this preprint (which was not certified by peer review) is the author/funder. All rights reserved. No reuse allowed without permission.

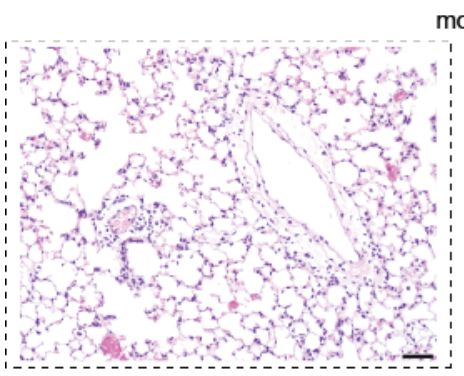

mock

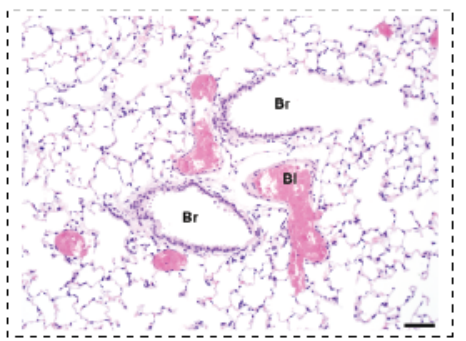

VOC $\delta$ - vehicle

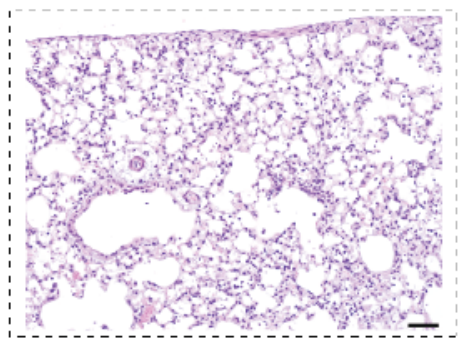

VOC $\delta$ - molnupiravir
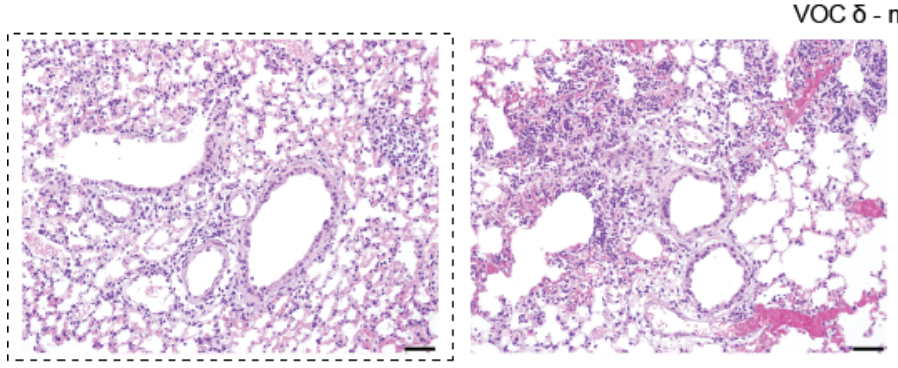

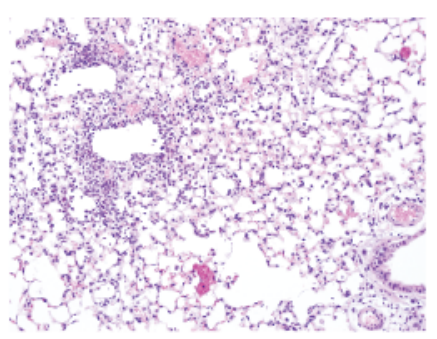

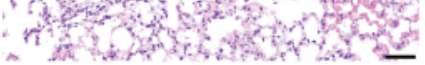

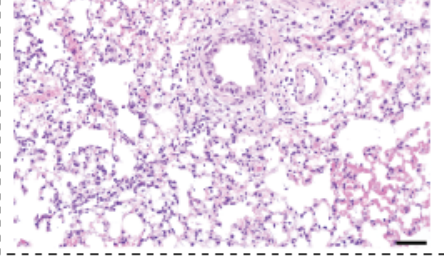

VOC $_{\mathrm{Y}}$ - vehicle

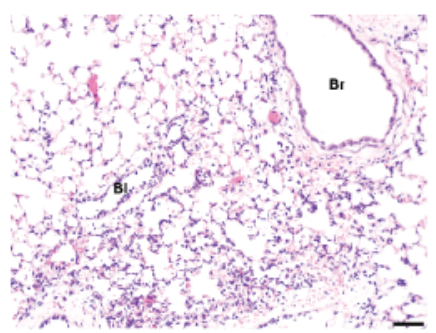

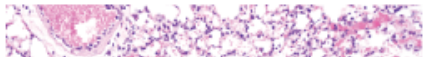
M. (f)

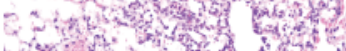

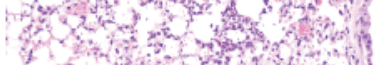

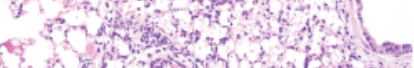

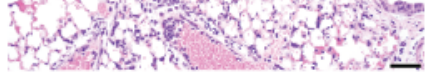
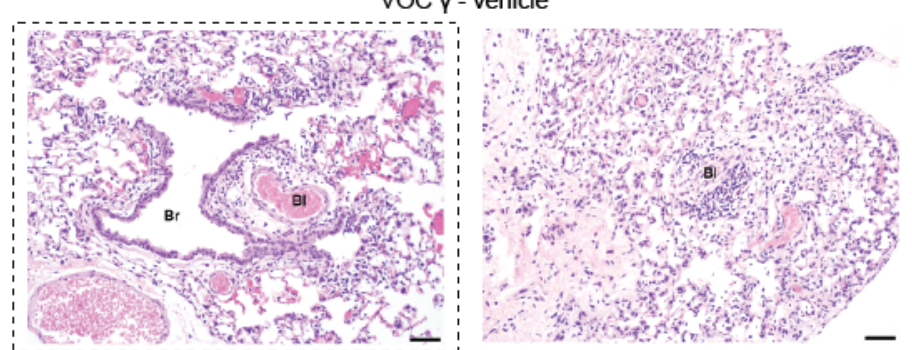

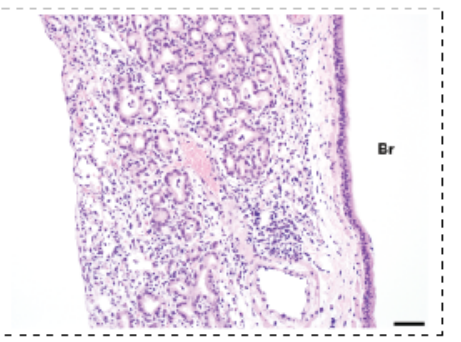

VOC $\mathrm{Y}$-molnupiravir
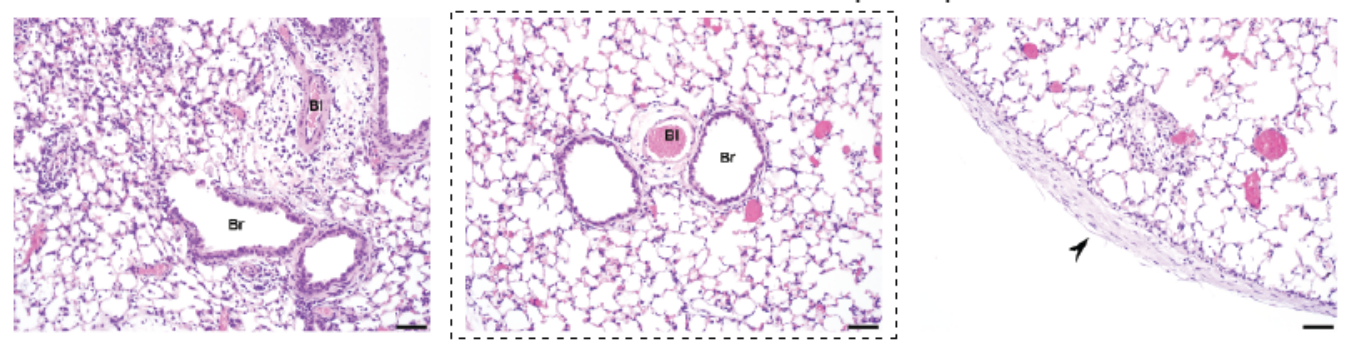
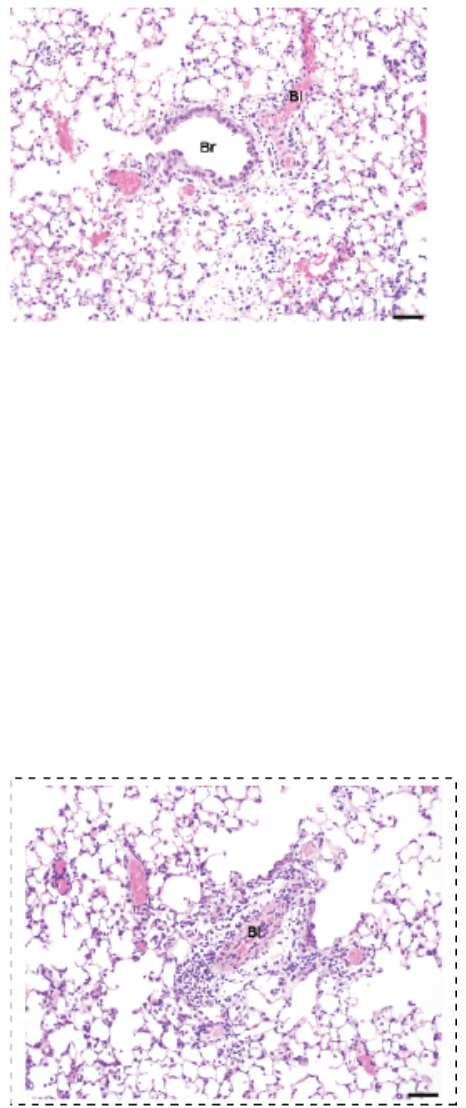

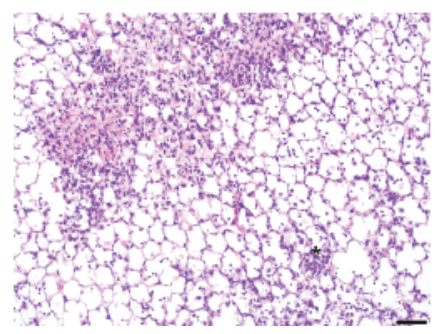


bioRxiv preprint doi: https://doi.org/10.1101/2022.02.04.479171; this version posted February 7, 2022. The copyright holder for this preprint (which was not certified by peer review) is the author/funder. All rights reserved. No reuse allowed without permission.

9 Extended Data 9. Histopathology of hamster lungs 3 days after infection. Staining with hematoxylin and eosin.

$0 \quad$ Arrows highlight pleuritis. Br, bronchioles; Bl, blood vessel; scale bar $50 \mu \mathrm{m}$.

VOC $\delta$ - vehicle treated - 14 days post infection
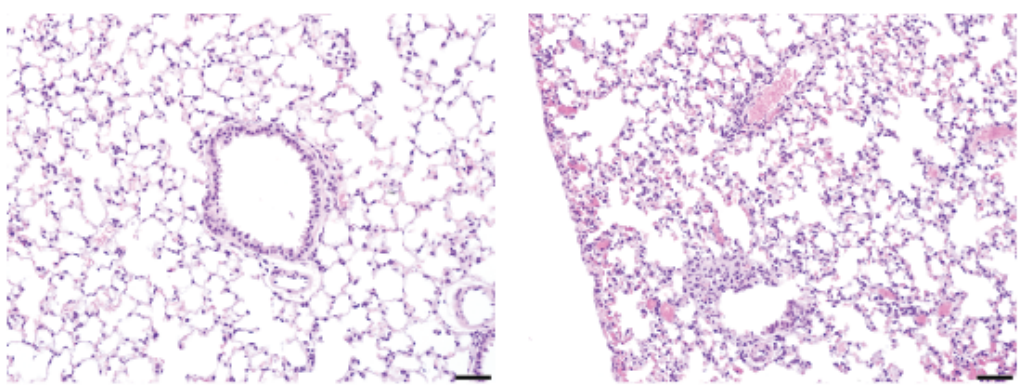

VOC $\delta$ - molnupiravir treated - 14 days post infection
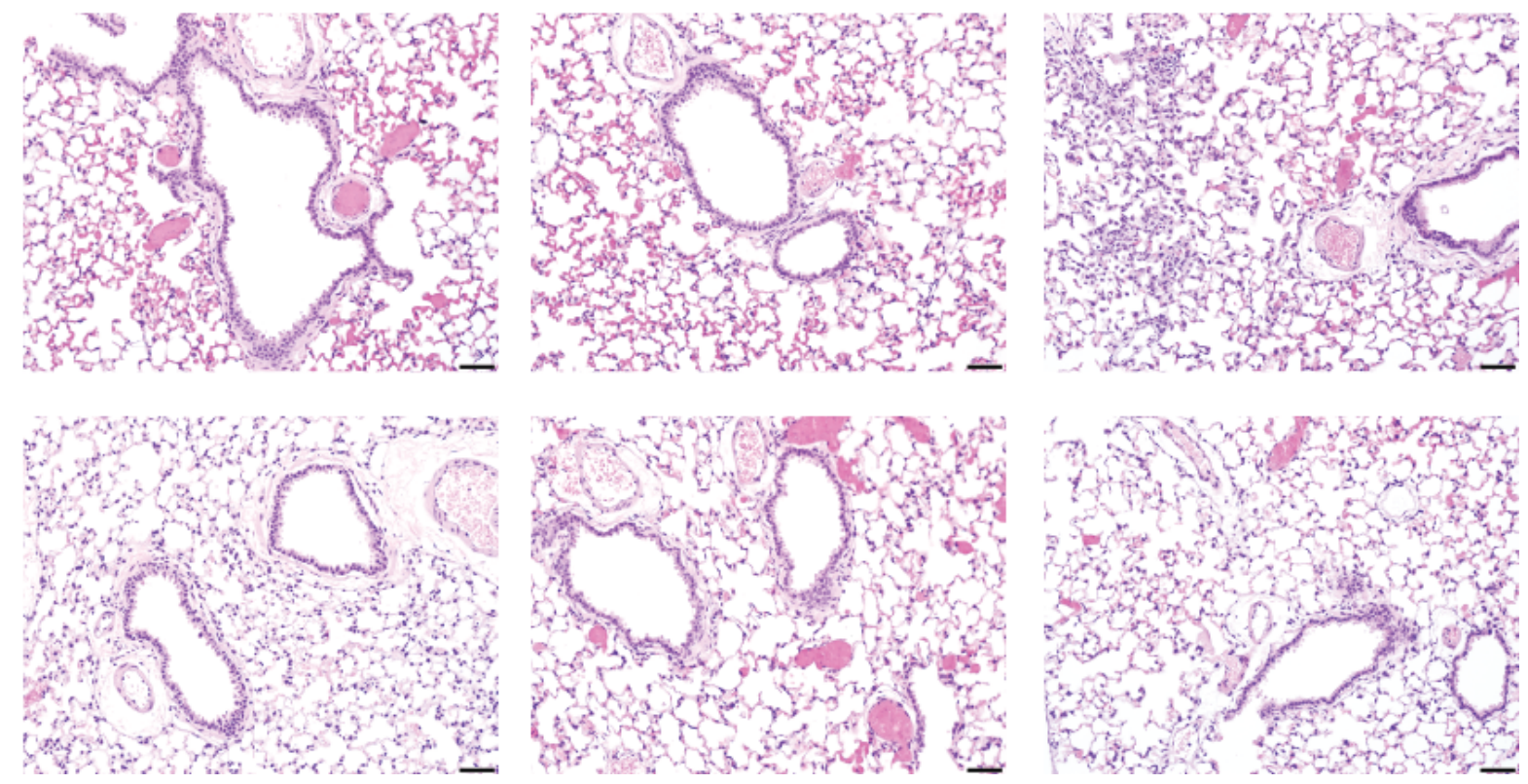

3 Extended Data 10. Histopathology of hamster lungs 14 days after infection. Staining with hematoxylin and 4 eosin. Scale bar $50 \mu \mathrm{m}$. 